\title{
THE TIDAL DISRUPTION OF NEUTRON STARS BY BLACK HOLES IN CLOSE BINARIES
}

\author{
JAMES M. LATTIMER \\ The University of Texas at Austin; and Enrico Fermi Institute, The University of Chicago \\ AND \\ DAVID N. SCHRAMM \\ Enrico Fermi Institute, The University of Chicago \\ Received 22 January 1976
}

\begin{abstract}
The formation of close, doubly compact binary systems from close massive binaries is considered. Massive X-ray binaries are shown to be possible progenitors of these systems. The recent observation of the binary pulsar indicates that doubly compact binary systems may exist. Reasonable scenarios can be developed in which at least one of the compact stars, in many cases, is a black hole. In general, these objects have evolved to separations less than $10 R_{\odot}$, and thus their orbits decay (within $10^{10} \mathrm{yr}$ ) via the emission of gravitational radiation. Black-holeneutron-star collisions may therefore be possible in these systems. A model for the tidal disruption of a neutron star by the black hole in these systems is constructed, and shows that mass ejection to infinity from $1.3 M_{\odot}$ neutron stars (a reasonable value suggested by evolutionary calculations) spiraling into black holes with masses less than $8-17 M_{\odot}$ is possible, this range depending upon the neutron star equation of state. For much larger black hole masses, the neutron star breakup occurs inside the Schwarzschild radius of the neutron star. A statistical estimate for the frequency of black-hole-neutron-star collisions is given, and possible implications for nucleosynthesis and antineutrino emission are discussed.
\end{abstract}

Subject headings: stars: binaries - black holes - stars: neutron

\section{INTRODUCTION}

The existence of binary X-ray sources can be satisfactorily explained in terms of compact, close binary systems (see Blumenthal and Tucker 1974 for a review). These sources are thought to be the result of the evolution of massive close binaries: the more massive star evolves first and "dies" in a supernovae explosion. Stellar evolutionary calculations as well as observational considerations (Arnett and Schramm 1973; Schramm and Arnett 1975; Tinsley 1975) imply that this supernova will produce a compact star (neutron star or black hole). (In fact, the massive $\mathrm{X}$-ray binary Cyg X-1 is thought to contain a compact star with a mass greater than $10 M_{\odot}$ [Paczynski 1974], which thus may be a black hole.) The X-ray source is then presumably powered by accretion of matter onto the compact star. If this supposition is correct, it implies that in at least some systems the supernova explosion leading to the formation of the compact star did not destroy the companion star or disrupt the binary. One may naturally inquire as to the subsequent fate of these systems. The discovery of the binary pulsar PSR 1913+16 (Hulse and Taylor 1975) has provided some impetus for such an investigation. Since the binary pulsar seems to consist of a neutron star together with a dwarf star or a compact star, evolutionary considerations (Flannery and van den Heuvel 1975) suggest that it may have originated from a massive X-ray binary. If, indeed, this system contains two compact stars, it is evidence that two supernovae may occur in a binary system without disrupting it.

This paper considers the probability and implication of the formation of doubly compact binary systems. If these binaries are formed with small enough separations, the emission of gravitational radiation will cause the orbit to completely decay, and the collision of the two components will result. There exist the following possible cases: (1) double black hole binary, (2) double neutron star binary, and (3) blackhole-neutron-star binary. In the first case the only observational consequence would be a prodigious emission of gravitational radiation (Smarr 1974), and this case is not considered further. The second is also not discussed in detail; however, some interesting possibilities exist, and they will be commented on. The third case has been considered by Lattimer and Schramm (1974) (hereafter referred to as LS). They conclude that the tidal forces exerted by the black hole are sufficiently strong to disrupt the neutron star before the two components actually collide (the "tube-oftoothpaste" mechanism - see Wheeler 1971). Furthermore, the breakup of the neutron star appeared to be violent enough to eject some neutron star matter completely out of the system. An estimate of the number of these events throughout the Galaxy indicated that perhaps a significant fraction of the neutron-rich nuclei in the Galaxy may be formed in 
this manner. The ejection of neutron-rich nuclei into the interstellar medium could have drastic consequences. For example, if a large amount of deuterium were formed, the current hypothesis that most deuterium is primordial in origin, and thus that the present density of matter is too low to close the Universe, might be in jeopardy (Epstein, Lattimer, and Schramm 1976). These interesting results warrant a more accurate investigation of this problem.

In $\S$ II, the formation of close doubly compact binaries is discussed. It is pointed out that mass transfer may greatly reduce the separations in these systems. Their orbits can then completely decay because of gravitational radiation in a time less than the age of the Universe, and the collision of the components will result. In $\S$ III, a model for a black-hole-neutron-star collision is constructed. First, the trajectory followed by the neutron star is analyzed. Then, the equations of hydrodynamics describing the neutron star in an inertial frame following this trajectory are developed. The tidal force exerted by the black hole is incorporated into these equations. The results of this model are discussed in $\S$ IV. The amount of mass ejected from the neutron star in the collision is determined, and the possible shortcomings of this model are reviewed. In $\S \mathrm{V}$ the observational implications of black-holeneutron-star collisions-nucleosynthesis, antineutrino radiation, and $\gamma$-ray bursts - are dealt with.

\section{THE FORMATION OF BINARIES CONTAINING TWO COMPACT STARS}

The initial stages of evolution of massive close binaries have been studied by several authors (e.g., Kippenhahn and Weigert 1967; Plavec 1968; Paczynski 1971). The present paper will be concerned only with binaries with initial separations less than a few AU, so that mass transfer is important. The more massive star (A) evolves faster; when its radius exceeds the mean radius $R_{\mathrm{R}}$ of the critical Roche surface, mass flows across the inner Lagrangian point onto its companion (B). Mass transfer causes A to lose its thermal equilibrium, but Morton (1960) has shown that readjustment to thermal equilibrium does not result in a decrease of the star's radius until the mass exchange becomes very large. Furthermore, if mass and angular momentum are conserved (reasonable assumptions since the mass flow occurs at velocities much less than escape velocities from either star), it is simple to show that, at least initially, the separation of the two stars must decrease. Since $R_{\mathrm{R}}$ is approximately given by Paczynski (1971) as

$R_{\mathrm{R}}=\left[0.38+0.2 \log \left(M_{\mathrm{A}} / M_{\mathrm{B}}\right)\right] a \quad\left(20>M_{\mathrm{A}} / M_{\mathrm{B}}>1\right)$

(where $M_{\mathrm{A}}$ and $M_{\mathrm{B}}$ are the masses of $\mathrm{A}$ and $\mathrm{B}$, and $a$ is the separation in the system), the size of the Roche surface also decreases, implying that further mass transfer is inevitable. The result is that nearly the entire envelope of A (about $70-80 \%$ of its mass) is generally transferred to $\mathrm{B}$, and A becomes a helium star.
It can be shown (Lattimer 1976) that only a negligibly small fraction of all binaries have masses $M_{\mathrm{A}}$ and $M_{\mathrm{B}}$ similar enough to allow the secondary, $\mathrm{B}$, to evolve significantly during either the mass transfer state or the further presupernova lifetime of A. It is generally known (see, e.g., Arnett and Schramm 1973) that helium stars more massive than about $4 M_{\odot}$ do not expand enormously in their later stages of evolution. Hence, if the original mass of $\mathrm{A}$ is greater than about $15 M_{\odot}$, the helium star left after the initial phase of mass exchange probably does not exceed its Roche surface in its future evolution; no further mass transfer from $\mathrm{A}$ is expected.

The models of massive stars of Arnett and Schramm (1973) indicate that stars in the range $6 \leqslant M / M_{\odot} \leqslant 70$ evolve to a similar presupernova configuration-a $1.4 M_{\odot}$ (approximately the Chandrasekhar mass) core with the rest of the mass contained in the mantle and envelope. Depending on the fraction of the mantle which falls on the core, a neutron star or black hole can be formed in a subsequent supernova explosion. (It may also be that a black hole could be formed with no associated supernova.) The effects of rotation and magnetic fields have been neglected in these calculations; it seems likely, though, that the general conclusion that a neutron star or black hole is formed is not altered. This consequence is supported observationally in the case of single stars, since (1) no white dwarfs are observed in open clusters with turnoff masses much greater than $6 M_{\odot}$, (2) the observed numbers of pulsars (presumably neutron stars) is consistent with the formation rate (and therefore the supernova rate) of single stars more massive than $\sim 6 M_{\odot}$, and (3) the supernova rate in galaxies is also consistent with this formation rate (Tinsley 1975).

In the case of binaries, the situation is more complicated. Mixing because of binary effects could lead to larger core masses and hence directly to black hole remnants. Such a possibility is suggested in the case of the massive X-ray binary Cyg X-1, where the collapsed component apparently has a mass greater than $\sim 10 M_{\odot}$. This binary, being one of the three massive X-ray sources within $3 \mathrm{kpc}$, therefore may indicate that black hole formation (with or without an accompanying supernova) in a binary system is not a rare event. (It is unlikely that the [presumed] black hole was formed by accretion onto a neutron star, since the companion has not yet evolved to its Roche lobe overflow stage.) The scenario outlined here may therefore lead to the formation of a collapsed star from the helium star A.

The existence of binary X-ray sources themselves suggests strongly that one supernova can occur in a close binary without disrupting it. Since B is now the more massive star, the mass ejected from $A$ in the supernova shell (if there indeed was a supernova) does not necessarily destroy B (Cheng 1974; Wheeler, McKee, and Lecar 1974) as only a small fraction of B $\left(\leqslant 0.01 M_{\mathrm{B}}\right)$ apparently is stripped off. This small amount of lost mass, however, reduces considerably the cross sectional area of B and is important in keeping the binary intact. Note the importance of the initial 
stage of mass transfer in keeping these binaries bound after the supernova explosion. Massive close binaries in this stage of evolution bear a strong resemblance to the models (see, e.g., Flannery and van den Heuvel 1975) of massive X-ray binaries. The X-rays may be powered by the accretion, onto the new compact star $A$, of the stellar wind emitted by its massive companion B. The theoretical considerations discussed above lead one to expect that these systems are likely to further evolve into (bound or unbound) doubly compact binaries. Two major evolutionary stages can be envisaged: First, the new primary (B), presumably a "normal" upper-main-sequence star, evolves and expands, entering a second Roche lobe overflow stage. Second, B may eventually become a supernova since its mass should exceed $6 M_{\odot}$ as a result of mass transfer from $A$.

In the first of these processes, for the reasons cited previously, most of the envelope of B is transferred in perhaps $10^{5} \mathrm{yr}$. However, since $\mathrm{A}$ is a compact star, it may not be able to accept most of this matter. Simply speaking, only if the accretion rate can exceed the rate permitted by the Eddington limit $\left(\sim 10^{-8} M_{\odot}\right.$ $\mathrm{yr}^{-1}$ ) by several orders of magnitude will all the transferred matter be accepted. Otherwise much of the matter may be lost from the system or perhaps a "double-core" star (Paczynski 1975) is formed. The Eddington limit refers to spherically symmetric accretion; in reality, the formation of an accretion disk (see, e.g., Shakura and Sunyaev 1973) and anisotropic accretion due to magnetic fields could raise the limit by perhaps two orders of magnitude. Neutrino cooling (Zel'dovich, Ivanova, and Nedezin 1972) could permit accretion rates as large as $10^{-1} M_{\odot} \mathrm{yr}^{-1}$, but recent calculations (Zytkov and Thorne 1974) imply that this is improbable. There is also the possibility that the unaccreted matter is not lost from the system, but is accreted gradually, long after the original mass transfer has stopped. The difficulty in ridding the accreting material of its angular momentum, however, makes accretion at rates much greater than $10^{-6} M_{\odot} \mathrm{yr}^{-1}$ seem physically unrealistic. Since the duration of mass transfer is $\leqslant 10^{5} \mathrm{yr}$, a compact star will be unable to accrete enough matter to form a black hole unless the Eddington limit is greatly exceeded or unless mass is accreted after the mass transfer has ended.

The second process occurs when B, now a helium star, becomes a supernova (or directly forms a black hole). The system is likely to be disrupted only if the mass loss $\Delta M$ from the supernova $\mathrm{B}$ is less than $\frac{1}{2}\left(M_{\mathrm{A}}+M_{\mathrm{B}}\right)$, assuming circular orbits, where now $M_{\mathrm{A}}$ and $M_{\mathrm{B}}$ refer to the presupernova masses. There are essentially two cases: (1) $M_{\mathrm{A}}$ is large because A formed a black hole either directly as a result of its evolution or because of high accretion rates or long time scales; (2) $\Delta M$ is small, because $M_{\mathrm{B}}$ is small or because $\mathrm{B}$ forms a black hole remnant with little mass ejection. The impact of the ejected supernova shell onto the compact star A with its correspondingly small cross section is probably not able, in itself, to disrupt the binary. Thus, systems in which at least one black hole is formed may be less likely to become disrupted. The existence of the binary pulsar (Hulse and Taylor 1975) is relevant here, since likely scenarios for its origin (Flannery and van den Heuvel 1975) suggest that this system has undergone at least one supernova, which has not caused the system's disruption. This system may be of rather small mass (see, e.g., Roberts, Masters, and Arnett 1976), perhaps only a few solar masses. It seems reasonable to suppose that more massive binaries may also remain bound.

A massive compact binary with a main-sequence primary (here, B) that has a mass in the range $2 \leqslant M_{\mathrm{B}} / M_{\odot} \leqslant 15$ may not be observable as an X-ray source (van den Heuvel 1975). Therefore, the number of observed massive X-ray binaries is a lower limit to the potential number of systems that may evolve into black-hole-neutron-star pairs. Massive X-ray binaries appear to be powered by the accretion of the stellar wind produced by B. This strong stellar wind persists only after core hydrogen burning has terminated and before Roche lobe overflow occurs. Thus de Loore et al. (1974) have estimated the lifetime of massive X-ray sources as (2-5) $\times 10^{4} \mathrm{yr}$. Therefore $3 /\left(\pi[3 \mathrm{kpc}]^{2} \times 5 \times 10^{4} \mathrm{yr}\right) \approx 2 \times 10^{-12} \mathrm{pc}^{-2} \mathrm{yr}^{-1}$ is a lower limit to the birthrate (in the galactic plane) of progenitors of doubly compact binaries, since there are observed to be three massive X-ray binaries within $3 \mathrm{kpc}$.

It has been argued that double compact binaries are likely to be formed by the further evolution of massive $\mathrm{X}$-ray binaries. The speculative nature of the arguments does not permit one to estimate very well the number of bound black-hole-neutron-star binaries that may result. The fact that one of the three massive X-ray binaries within $3 \mathrm{kpc}$, Cyg X-1, is currently thought to contain a black hole is evidence that the first supernova explosion in this system produced the black hole, as the second phase of mass exchange has not yet begun. If Cyg X-1 is any indication, doubly compact binaries with at least one component a black hole may be relatively common; it may be that the second supernova explosion does not disrupt these systems because of the mass in the collapsed component. Observationally, one expects these systems to be difficult to detect: unless one of the compact stars is a pulsar, or accretion is occurring, these binaries will be completely "dark." Van den Heuvel (1975) has argued that if all massive X-ray binaries evolved into systems containing a pulsar as a result of a second supernova explosion, there would be $\frac{3}{5} \times$ (pulsar lifetime $) /(\mathrm{X}$-ray lifetime $) \approx$ 60 observable binary pulsars within $3 \mathrm{kpc}$, if one-fifth (due to beaming) of pulsars are observable, and if a pulsar lifetime is about $4 \times 10^{6}$ yr. Because Cyg X-1 may contain a black hole, a smaller, but presumably nonzero, number of pulsars formed with black hole companions might be expected.

A pulsar created in a system with a companion star which is not massive might resemble the binary pulsar PSR $1913+16$. This type of system may, however, be formed unbound in many instances, leading to the observed deficiency of these binaries. The observed lack of pulsars in systems containing a massive 
collapsed object, on the other hand, may be due to the disruption of the system by the second supernova explosion. Or, the pulsing mechanism of the new supernova remnant may be masked or destroyed by the presence of the black hole. The latter cause might be compared with what happens if a neutron star is formed in a system containing a massive mainsequence star: the pulsar may be converted into an $\mathrm{X}$-ray source. The disruption of binary pulsars by supernova explosions is not inconsistent with the observed numbers of single pulsars or their velocity distributions. However, the typical pulsar velocity observed, which is of the order of a few hundred $\mathrm{km} \mathrm{s}^{-1}$, could instead be explained by a slightly asymmetric supernova explosion in single stars.

Black-hole-neutron-star binaries, if they are formed, would be of great interest because of their initial proximity. The various processes of mass transfer and mass loss in close binaries appear to lead to a significant reduction in the separation of the components. The most important stage in this consideration is the second phase of mass transfer, from B to the collapsed star A. If all the accreted matter is accepted, so that mass and orbital angular momentum are conserved, the initial (subscript 0) and final (subscript $f$ ) separations are related by

$$
a_{f}=\left(\frac{M_{\mathrm{A} 0} M_{\mathrm{B} 0}}{M_{\mathrm{Af}} M_{\mathrm{Bf}}}\right)^{2} a_{0} .
$$

For example, if $M_{\mathrm{B} 0}=20 M_{\odot}, M_{\mathrm{A} 0}=1 M_{\odot}$, and $M_{\mathrm{Bf}} \approx 5 M_{\odot}$, then $M_{\mathrm{Af}}=16 M_{\odot}$ and $a_{f} / a_{0} \approx 1 / 16$. If, instead, mass was lost from the system, assuming the orbital energy to be conserved and $M_{\mathrm{B} 0} \gg M_{\mathrm{A} 0}$, one has $a_{f}=\left(M_{\mathrm{B} f} / M_{\mathrm{B} 0}\right) a_{0}$. Once again a large reduction in the separation is implied. In the latter case, a more realistic assumption is that part of the dynamical energy of the binary is required to expel the mass from the system. The reduction is then even larger (van den Heuvel and de Loore 1973), varying exponentially with $M_{\mathrm{B} f}-M_{\mathrm{B} 0}$. Compared with these factors, expansion of the orbit in the supernova processes is negligible.

The emission of gravitational radiation will cause the black-hole-neutron-star pair to slowly spiral together. If the separation with which they are formed satisfies (see, e.g., Misner, Thorne, and Wheeler 1973)

$$
a<2.8[M m(M+m)]^{1 / 4} R_{\odot},
$$

where $M$ and $m$ are the black hole and neutron star masses in solar units, the orbit will completely decay within the age of the Universe $\left(\sim 10^{10} \mathrm{yr}\right)$. Choosing as typical values $M=10$ and $m=1$, equation (3) gives $a<9 R_{\odot}$. This implies that the initial orbit must be less than $100-200 R_{\odot}$ in order for the separation to decrease to the required value by the time the doubly compact binary is formed. Massive X-ray binaries apparently have separations less than these values.

In conclusion, massive close binaries may evolve into black-hole-neutron-star or other doubly compact systems. It seems reasonable that the massive X-ray binaries are one stage in this evolution and therefore can be used to estimate the potential number of close systems containing two collapsed stars. The second stage of mass transfer may be generally capable of reducing the separations in these systems enough to allow gravitational radiation to become important. Hence, a black-hole-neutron-star collision appears probable. In $\S$ III, we construct a detailed model for this interaction.

\section{MODEL FOR BLACK-HOLE-NEUTRON-STAR COLLISIONS}

In order to make the problem tractable it will be assumed that the neutron star mass $m$ is much smaller than the black hole mass $M$. The black hole is assumed to be nonrotating. (For the extension to the case of a rotating hole, see Lattimer 1976.) The trajectory of the neutron star is then determined by the background Schwarzschild geometry (geometric units $c=G=1$ are used throughout)

$$
\left(\frac{d R}{d \tau}\right)^{2}=\gamma^{2}-X\left(1+\frac{l^{2}}{R^{2}}\right),
$$

where $X=1-2 M / R, R$ is the radial coordinate, $\tau$ is the proper time, $\gamma$ is the energy per unit rest mass at infinity of the neutron star, and $l$ is the orbital angular momentum per unit rest mass of the neutron star. The orbit is assumed to be nearly circular at radial infinity, but the emission of gravitational radiation causes the trajectory (initially) to be slowly spiraling. The weakfield approximation (Landau and Lifshitz 1971) gives the orbital energy loss rate to a good approximation (Lattimer 1976) outside of $R=6 M$. For circular orbits this rate is

$$
\frac{d \gamma}{d \tau}=-6.4 M^{2} m(M+m) R^{-5}
$$

In general, the orbital angular momentum loss rate will be given by the "universal energy-angular momentum-relation" (e.g., Page and Thorne 1974),

$$
\frac{d l}{d \tau}=\frac{d \gamma}{d \tau} \Omega^{-1}
$$

where the orbital frequency $\Omega$ is $l X \gamma^{-1} R^{-2}$ for circular orbits. By differentiating equation (4) twice with respect to the proper time, one finds (after making use of eq. [6])

$$
\begin{aligned}
\frac{d^{3} R}{d \tau^{3}}= & \frac{2 \gamma}{R X} \frac{d \gamma}{d \tau}(1-3 M / R)-\frac{3}{R^{3}} \frac{d R}{d \tau}(1-4 M / R) \\
& \times\left(R^{2} \frac{d^{2} R}{d \tau^{2}}+M\right)(1-3 M / R)+\frac{2 M}{R^{3}} \frac{d R}{d \tau}
\end{aligned}
$$

The energy $\gamma$ may be eliminated from equation (7) by differentiating equation (4) and using equation (6):

$$
\gamma^{2}=\left(\frac{d R}{d \tau}\right)^{2}+X\left(X+R \frac{d^{2} R}{d \tau^{2}}\right)(1-3 M / R)^{-1}
$$

To solve equation (7), one requires initial conditions 
for $R, d R / d \tau$, and $d^{2} R / d \tau^{2}$. For consistency in evaluating the initial conditions for $d R / d \tau, d^{2} R / d \tau^{2}$ and $d^{3} R / d \tau^{3}$ are assumed for some large $R$ to be given by twice and thrice differentiating the Schwarzschild relation

$$
\gamma=X(1-3 M / R)^{-1 / 2}
$$

and using equation (5) for $d \gamma / d \tau$. Then the cubic equation derived from equation (7) for $d R / d \tau$ is solved; it is found that this initial value for $d R / d \tau$ is extremely close to that given by differentiating once equation (9) and using equation (5) for $d \gamma / d \tau$. Having initial values for $R, d R / d \tau$, and $d^{2} R / d \tau^{2}$, one may now integrate equation (7).

This method of calculating the spiral trajectories differs from that used by LS; they used the simpler Newtonian relation $\gamma=1-M / 2 R$ to calculate $d R / d \tau$ from equation (5). Thus

$$
\left(\frac{d R}{d \tau}\right)_{\mathrm{LS}}=-12.8 M m(M+m) R^{-3}
$$

Figure 1 shows the rates $d R / d \tau$ predicted by these two methods.

It is clear that formula (10) severely underestimates these rates for $R \leqslant 10 M$-this has effects on the results, to be discussed later.

Equation (7) for the neutron star's trajectory will be only as valid as the approximation that $(m / M) \ll 1$.

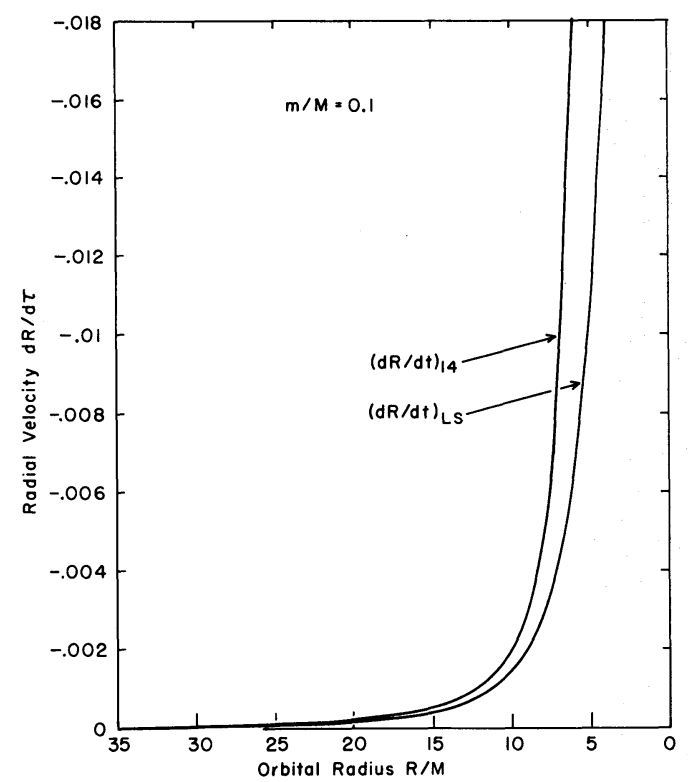

FIG. 1:- The radial velocity of the center of mass of the neutron star as it spirals toward the black hole. Velocity is in units of $c$; the radial coordinate $R$ is in units of the black hole mass $M$. The quantity $(d R / d \tau)_{\mathrm{LS}}$ is the radial velocity used by Lattimer and Schramm (1974) (eq. [10]); the curve representing the integration of eq. (7) is denoted by $(d R / d \tau)_{13}$.
In order that the neutron star's spin not affect its trajectory, Corinaldesi and Papapetrou (1951) show that

$$
\frac{M}{R} \frac{r^{2}}{R^{2}} \frac{\Omega^{-1}}{\omega^{-1}} \ll 1
$$

is required, where $r$ is the radius of the neutron star and $\omega$ its rotational frequency. Even if the neutron star is rotating near breakup velocity, that is, when $\omega \sim\left(m r^{-3}\right)^{1 / 2}$, this inequality reads

$$
\left(\frac{M}{R} \frac{m}{R} \frac{r}{R}\right)^{1 / 2} \ll 1,
$$

where the approximate formula $\Omega \sim\left(M R^{-3}\right)^{1 / 2}$ has been used. For $R \sim 6 M \sim 6 r$ and $m / M \sim 0.1$ (which will be seen to be typical values), this is a good approximation.

In order to study the neutron star as it is deformed in the tidal field of the black hole, a frame of reference is needed. In general relativity the closest analog to an inertial frame is a Fermi-Walker transported tetrad frame (Misner, Thorne, and Wheeler 1973). This Fermi frame consists of an orthonormal tetrad of basis vectors $e_{(\mu)}$ (indices referring to the Fermi frame will be enclosed in parentheses; $\mu, \nu=0,1,2,3$; and $i, j=$ $1,2,3)$ such that $e_{(\mu)} \equiv \partial / \partial X^{(\mu)}$ and $e_{(\mu)} \cdot e_{(v)}=\eta_{\mu v}$. Thus, the components $e_{(\mu)}^{v}$ of these vectors form the transformation correct to order $\left(X^{(\mu)} / X^{\mu}\right)$ between the Schwarzschild $\left(X^{\nu}=(T, R, \Theta, \Phi)\right)$ and the Fermi $\left(X^{(\mu)}\right)$ frames:

$$
e_{(\mu)}^{\nu}=\frac{\partial X^{\nu}}{\partial X^{(\mu)}}
$$

Since the Fermi frame is parallelly transported along the orbit, one has

$$
\frac{d e^{\nu}{ }_{(\mu)}}{d \tau}+e^{\omega}{ }_{(0)} e^{\lambda}(\mu) \Gamma_{\omega \lambda}^{\nu}=-e_{(\omega)}^{\nu} \Omega^{(\omega)}{ }_{(\mu)},
$$

where the $\Gamma^{\alpha}{ }_{\mu v}$ are the Christoffel symbols in the Schwarzschild frame and $\Omega^{(\omega)}{ }_{(v)}$ is the infinitesimal generator of Lorentz transformations; see Misner, Thorne, and Wheeler (1973) for details. Choose

$$
e_{(0)}=\left(\frac{d T}{d \tau}, \frac{d R}{d \tau}, \frac{d \Theta}{d \tau}, \frac{d \Phi}{d \tau}\right),
$$

and assume the orbit to lie in the plane $\Theta=\pi / 2$ so that $e_{(2)}=\left(0,0, R^{-1}, 0\right)$. The general solution of equation (13) can be written as

$e_{(1)}=(A \cos \phi+D \sin \phi, B \cos \phi+E \sin \phi, 0, F \sin \phi)$,

$e_{(3)}=(D \cos \phi+A \sin \phi, E \cos \phi+B \sin \phi, 0, F \cos \phi)$. 
The solution of equation (13) in the case of geodesic motion $\left(\Omega_{(\omega)}^{(\omega)}=0\right)$ in the Schwarzschild metric gives (Matzner and Nutku 1974), using $N=\left(1+l^{2} / R^{2}\right)^{1 / 2}$,

$$
\begin{aligned}
& A=\left(\frac{d R}{d \tau}\right) / N X ; \quad B=\gamma / N ; \quad D=\gamma l / X R N ; \\
& E=\left(\frac{d R}{d \tau}\right) l / R N ; \quad F=N / R,
\end{aligned}
$$

and

$$
d \phi / d \tau=-\gamma l /\left(R^{2} N^{2}\right) .
$$

In the case to be considered, the acceleration by the emission of gravitational radiation causes the trajectory of the neutron star to deviate from a geodesic. One can show that a nonzero $\Omega^{(\omega)}{ }_{(v)}$ leads to a modified equation for $\phi$ :

$$
\frac{d \phi}{d \tau}=-\gamma l\left[1+R\left(a^{0} \frac{d R}{d \tau} / \gamma-\frac{a^{1}}{X}\right)\right] /\left(R^{2} N^{2}\right),
$$

where the acceleration $a^{\alpha}$ is given by

$$
a^{\alpha}=\frac{d^{2} X^{\alpha}}{d \tau^{2}}+\Gamma_{\mu \nu}^{\alpha} \frac{d X^{\mu}}{d \tau} \frac{d X^{\nu}}{d \tau}
$$

Since the weak field approximation for the decay of the orbit via gravitational radiation gives $d \gamma / d \tau \sim$ $(M / R)^{5}$ and (for Newtonian circular orbits) $d R / d \tau \sim$ $(M / R)^{3}$ and $d^{2} R / d \tau^{2} \sim(M / R)^{7}$, the additional term in equation (19) is of order $(M / R)^{6}$ (note that $a^{0}=$ $(d \gamma / d \tau) / X$ and $\left.a^{1} \sim d^{2} R / d \tau^{2}\right)$. This term is negligible since, for the Fermi frame analysis to be valid, the inequality $M / R \ll 1$ (see eq. [36] with $X^{(i)} \sim M$ ) must hold.

The Fermi tetrad can be viewed, at least locally, as a Newtonian inertial frame. Insofar as the neutron star material is nonrelativistic and the fluid velocities are much less than $c$, one may then approximate the equations of hydrodynamics in this frame by the standard Euler equations:

$$
\begin{gathered}
\rho \frac{d^{2} X_{i}}{d \tau^{2}}=-\frac{\partial P}{\partial X_{i}}+\rho \frac{\partial U}{\partial X_{i}}+\rho \frac{\partial \Theta}{\partial X_{i}}, \\
\frac{\partial \rho}{\partial \tau}+\frac{\partial}{\partial X_{i}}\left(\rho \frac{d X_{i}}{d \tau}\right)=0 .
\end{gathered}
$$

For simplicity the Fermi coordinates have been written as $X^{(\mu)}=\left(\tau, X_{i}\right)$; and $P, \rho$, and $U$ are, respectively, the pressure, density, and the self-gravitational potential of the material under consideration. Summation over repeated indices is implied. $\Phi$ is an external tidal potential acting on the material and will be specified later. The effects of viscosity have been neglected.

The usual method of solution of the Euler equations (in the absence of tidal forces) for a constant-density perfect fluid constrained to an ellipsoidal shape is outlined in Chandrasekhar (1969). The calculations are done with respect to the rest frame of the ellipsoid. The results of LS were obtained using this model to represent the neutron star, but included tidal forces. However, when dealing with configurations under the influence of tidal forces or viscosity in which the two moments of inertia perpendicular to the rotation axis can be equal, singularities in the solutions appear (see the Appendix). One way to avoid this difficulty is to begin the integration close enough to the black hole that the ellipsoid is deformed sufficiently to ensure that the two moments of inertia perpendicular to the rotation axis never become equal. This procedure, however, may influence the results obtained. Therefore, a method of solving these equations in the inertial frame is developed here which eliminates these singularities.

The first approximation to be introduced is the standard assumption that the internal velocities are a linear function of the coordinates:

$$
\frac{d X_{i}}{d \tau}=Q_{i j}(\tau) X_{j}
$$

Equation (21) becomes, after substituting (23), multiplying by $X_{j}$, and integrating over the volume $V$ occupied by the fluid,

$$
\frac{d Q_{i k}}{d \tau} I_{k j}=-Q_{i k} Q_{k l} I_{l j}+\Pi \delta_{i j}+B_{i j}-R_{i j}
$$

Here, using $I_{0}=m / 5$ with $m$ the mass of the ellipsoid,

$$
\begin{aligned}
I_{i j} & =\int_{V} \rho X_{i} X_{j} d V / I_{0}, \\
\Pi \delta_{i j} & =-\int_{V} X_{j} \frac{\partial P}{\partial X_{i}} d V / I_{0}, \\
B_{i j} & =\int_{V} \rho X_{j} \frac{\partial U}{\partial X_{i}} d V / I_{0},
\end{aligned}
$$

and

$$
R_{i j}=-\int_{V} \rho X_{j} \frac{\partial \Phi}{\partial X_{i}} d V / I_{0}
$$

$B_{i j}$ is more easily calculated in the rest frame, rather than the inertial frame of the ellipsoid. One can then obtain its inertial frame value by a transformation applied to its value in the rest frame (denoted by primes):

$$
B_{i j}=T_{l i} B^{\prime} T_{k j} .
$$

$T_{i j}$ is the usual rotation matrix (assuming the rotation to lie only in the " $1-3$ " plane):

$$
T=\left(\begin{array}{ccc}
\cos \theta & 0 & \sin \theta \\
0 & 1 & 0 \\
-\sin \theta & 0 & \cos \theta
\end{array}\right) \text {, }
$$

with $-d \theta / d \tau=\Omega$, the angular velocity (assumed to lie in the " 2 " direction). This transformation is valid for 
any matrix. By definition $I^{\prime}{ }_{i j}$ is diagonal, and $\theta$ can be determined from $I^{\prime}{ }_{13}=0$; from (29) and (30) one has

$$
\theta=\frac{1}{2} \tan ^{-1}\left[\frac{2 I_{13}}{I_{11}-I_{33}}\right] \text {. }
$$

Equation (25) leads to the identity

$$
\frac{d I_{i j}}{d \tau}=Q_{i k} I_{k j}+Q_{j k} I_{k i}
$$

which will later prove to be useful. $Q_{i j}$ contains two nonzero off-diagonal elements, $Q_{13}$ and $Q_{31}$. One of these can be eliminated as follows: Define the circulation $C$ around a closed path $l$ as

$$
C=\oint \frac{d X}{d \tau} \cdot d l
$$

so that

$$
C=\int_{\partial V}\left(\nabla \times \frac{d X}{d \tau}\right)_{2} d S_{2}=\pi a_{1} a_{3}\left(Q_{31}-Q_{13}\right),
$$

where $d S$ is an element of the surface $\partial V$ and $a_{1} a_{3}$ is the area of the ellipsoid projected onto the equatorial plane. In the absence of viscosity, equation (24) can be manipulated to show that $C$ is conserved. Thus, either $Q_{13}$ or $Q_{31}$ may be eliminated.

It still remains to specify the tidal potential $\Phi$. The equation of geodesic deviation (Misner, Thorne, and Wheeler 1973) details how the perturbing field of the black hole affects the motion of particles lying near the center of the Fermi frame. In the case of the Fermi frame being transported along a geodesic, $\Phi$ is, to first order (Pirani 1956),

$$
\Phi=-\frac{1}{2} R_{(i)(0)(j)(0)} X^{(i)} X^{(j)},
$$

where $R_{(\alpha)(\beta)(\mu)(v)}$ lists the components of the Riemann tensor in Fermi coordinates, evaluated at the center of the Fermi frame. Thus,

$$
R_{(i)(0)(j)(0)}=e_{(i)}^{\mu} e_{(0)}^{v} e_{(j)}^{\omega} e_{(0) \lambda} R^{\lambda}{ }_{\mu \nu \omega},
$$

where $R^{\lambda}{ }_{u v \omega}$ is the Riemann tensor in Schwarzschild coordinates. Equation (35) is valid only when the coordinates $X^{(i)}$ satisfy

$$
X^{(i)} \ll \mid \text { average value of Riemann tensor }\left.\right|^{-1 / 2} \text {, }
$$

and when $d X^{(i)} / d \tau \ll 1$. If the latter condition is not satisfied, equation (35) can be written in the form of a power series in $d X^{(i)} / d \tau$ (Mashhoon 1975):

$$
\begin{aligned}
\Phi=-\frac{1}{2}[ & R_{(i)(0)(j)(0)}+2 R_{(i)(k)(j)(0)} \frac{d X^{(k)}}{d \tau} \\
+2 R_{(0)(k)(j)(0)} & \frac{d X^{(i)}}{d \tau} \frac{d X^{(k)}}{d \tau}+\frac{2}{3} R_{(i)(k)(j)(l)} \\
& \left.\times \frac{d X^{(k)}}{d \tau} \frac{d X^{(l)}}{d \tau}\right] X^{(i)} X^{(j)} .
\end{aligned}
$$

Since, for the Schwarzschild geometry, $R_{(i)(k)(j)(0)}=0$, the correction enters only to second order in $d X^{(i)} / d \tau$, and hence is negligible until the breakup of the neutron star is well underway. Similarly, the effect of accelerations caused by pressure and binding forces within the neutron star and the orbital accelerations due to the emission of gravitational radiation enter as the time derivative of the acceleration (Mashhoon 1975) and are negligible. The largest error in assuming equation (35) is that the Fermi frame extends over a finite extent: equation (35) should therefore be viewed as the leading term in a power series in $X^{(i)}$ for $\Phi$. A second order correction to (35) would not contribute to $R_{i j}$ (eq. [28]) since odd powers of $X^{(i)}$ integrated over the mass of an ellipsoid are zero; higher order terms are neglected.

If it is assumed that $\Phi$ is given by equation (35), a simple calculation gives for equation (28)

$$
R_{i j}=R_{(i)(0)(k)(0)} I_{k j} \text {. }
$$

Most of the mass of a moderately sized neutron star is at roughly the same density. A simple first approximation to a neutron star is a constant-density fluid constrained to an ellipsoidal shape. Equation (22) implies, for $\rho=$ constant, that $Q$ is traceless. For this case, Chandrasekhar (1969), for example, gives $B^{\prime}{ }_{i j}$ as

$$
B_{i j}^{\prime}=-2 \pi \rho \delta_{i j} A_{i} a_{i}^{2},
$$

where the $a_{i}$ are the semiaxes of the ellipsoid as viewed in the rest frame and the $A_{i}$ are functions of only the $a_{i}$ and are described in Chandrasekhar (1969), pp. 4143. The $a_{i}$ can be found from the $I^{\prime}{ }_{i j}$ :

$$
I_{i j}^{\prime}=\delta_{i j} a_{i}^{2} .
$$

The $I^{\prime}{ }_{i j}$ are found from $I_{i j}$ by equation (29). Since the fluid is incompressible, the volume of the ellipsoid is constant, so the product $I^{\prime}{ }_{11} I_{22}^{\prime} I_{33}^{\prime}=$ constant-see also equation (A23). Thus $\Pi$ can be eliminated from (24). Equations (24), (32), (6)-(8), and (19), together with the constraints of incompressibility and conservation of circulation (eq. [34]), provide a complete description of the evolution of the system.

Though this model is useful for the delineation of the major aspects of black-hole-neutron-star collisions, a more realistic neutron star model is also considered. A large fraction of the neutron star's volume is at lower densities than the bulk of its mass. The less dense portions of the neutron star, being less bound, are more apt to be ejected in the neutron star breakup. In a more realistic model, the assumption that the fluid is incompressible may be removed by providing an equation of state of the form

with

$$
P(X, \tau)=\eta \rho^{\Gamma}(X, \tau),
$$

$$
\rho(X, \tau)=\rho_{c}(\tau)\left(1-p^{2}\right)^{n(X, \tau)},
$$

where $\rho_{c}(\tau)$ is the central density, and $n, \eta$ and $\Gamma$ are to be determined. It is assumed that the ellipsoid is made up of shells, each interior shell having the same ratios of semiaxes $\left(p a_{1}: p a_{2}: p a_{3}\right)$ as the surface $\left(a_{1}: a_{2}: a_{3}\right)$. Here $p^{2}=\sum_{i} X_{i}^{2} / a_{i}^{2}$ is a constant on the 
surface of each shell and represents a normalized "radial" coordinate. The mass contained within each shell is therefore constant; no mass may flow from shell to shell. The requirement that $p$ be a constant implies

$$
\frac{d p}{d \tau}=\frac{\partial p}{\partial \tau}+\frac{d X_{i}}{d \tau} \frac{\partial p}{\partial X_{i}}=0 .
$$

Thus (summation over $i$ and $j$ implied)

$$
X_{i}^{2} a_{i}^{-3} d a_{i} / d \tau=X_{i} a_{i}{ }^{-2} d X_{i} / d \tau=X_{i} a_{i}^{-2} Q_{i j} X_{j} .
$$

Equation (22) now gives

$$
\begin{gathered}
\frac{d \rho_{c}}{d \tau} \frac{\rho}{\rho_{c}}+2 n \rho_{c}\left(1-p^{2}\right)^{n-1}\left(X_{k}^{2} a_{k}{ }^{-3} \frac{d a_{k}}{d \tau}-Q_{k j} X_{j} X_{k} a_{k}^{-2}\right) \\
+\rho \ln \left(1-p^{2}\right)\left(\frac{\partial \eta}{\partial \tau}+\frac{\partial n}{\partial X_{k}} Q_{k j} X_{j}\right)+\rho \sum_{i} Q_{i i}=0 .
\end{gathered}
$$

Evaluating (45) at $X_{1}=X_{2}=X_{3}=0$, one finds

$$
\frac{d \rho_{c}}{d \tau}+\rho_{c} \sum_{i} Q_{i i}=0 \text {. }
$$

Substituting (44) and (46) into (45) yields

$$
\frac{d n}{d \tau} \equiv\left(\frac{\partial n}{\partial \tau}+\frac{\partial n}{\partial X_{k}} Q_{k j} X_{j}\right)=0 .
$$

Thus $n=n(p)$ may be chosen initially to fit equation (42) to a tabulated model; thereafter, it remains constant. To simplify the model more, without loss of generality one may choose $\eta$ and $\Gamma$ to be functions of $\rho$; they too are selected initially to fit equation (41) to a tabulated model. In what follows, the notation $\eta(p)$ means the value of $n(\bar{\rho})$ in the $p$ th shell where the mean density is $\bar{\rho}$. tion

Roberts (1962) has shown that in terms of the func-

$$
F_{n}\left(p^{2}\right)=\int_{p^{2}}^{1}\left(1-p^{2}\right)^{n(p)} d p^{2},
$$

the functions $I^{\prime}{ }_{i j}$ and $B^{\prime}{ }_{i j}$ can be written as

$$
I_{i j}^{\prime}=2 \pi \rho_{c} I_{0}{ }^{-1} a_{1} a_{2} a_{3} \delta_{i j} a_{i}{ }^{2} \int_{0}^{1} p^{2} F_{n}\left(p^{2}\right) d p
$$

and

$$
B^{\prime}{ }_{i j}=\pi^{2} \rho_{c}^{2} I_{0}{ }^{-1} a_{1} a_{2} a_{3} A_{i} \delta_{i j} a_{i}^{2} \int_{0}^{1}\left[F_{n}\left(p^{2}\right)\right]^{2} d p .
$$

If the pressure vanishes on the surface of the neutron star, equation (26) may be written as

$$
\begin{aligned}
\Pi & =\frac{1}{I_{0}} \int_{V} P d V \\
& =4 \pi a_{1} a_{2} a_{3} I_{0}{ }^{-1} \int_{0}^{1} \eta(p) \rho_{c}^{\Gamma(p)}\left(1-p^{2}\right)^{n(p) \Gamma(p)} p^{2} d p .
\end{aligned}
$$

Denoting the $\sigma$ th shell as bounded by $p_{\sigma-1}$ and $p_{\sigma}$, define

$$
G_{\sigma}(n)=\int_{p_{\sigma-1}}^{p_{\sigma}}\left(1-p^{2}\right)^{n\left(p_{\sigma}\right)} p^{2} d p .
$$

Then, assuming for simplicity that $\eta$ and $\Gamma$ are constant within each shell,

$$
\Pi=I_{0}{ }^{-1} 4 \pi a_{1} a_{2} a_{3} \sum_{\sigma} \eta\left(p_{\sigma}\right) \rho_{c} \Gamma^{\left(p_{\sigma}\right)} G_{\sigma}(n \Gamma) .
$$

Note that the mass of the ellipsoid can be written as

$$
m=5 I_{0}=2 \pi \rho_{c} a_{1} a_{2} a_{3} \int_{0}^{1} F_{n}\left(p^{2}\right) d p,
$$

showing that $\rho_{c} a_{1} a_{2} a_{3}=$ constant since $d p / d \tau=$ $d n / d \tau=0$.

The neutron star models were computed from the tabulated models of Arnett and Bowers (1974). The function $n(p)$ calculated for spherical stars from equation (42) remains valid for arbitrary ellipticity if $d n / d \tau=d p / d \tau=0$, as has been assumed. The model star is divided into 25 concentric shells; the shells are chosen to contain equal mass: the results are not sensitive to this choice. From the equations of state tabulated in Arnett and Bowers (1974), the functions $\eta(p)$ and $\Gamma(p)$ are determined. The functions $F_{n}\left(p^{2}\right)$ and $G_{\sigma}(n)$ can be computed from equations (48) and (52) once and for all. For nonspherical stars, $\eta(p)$ and $\Gamma(p)$ are calculated by finding the value of the average density $\bar{\rho}$ corresponding to the $p$ th shell; it is assumed that $\eta$ and $\Gamma$ are then given by the original equation of state through equation (41).

In what is to come, a technique is required for determining whether or not the infalling neutron star is deformed rapidly enough to enable part of it to be ejected. Certainly when deviations from sphericity are pronounced, the constraint that the neutron star material have an ellipsoidal shape will be in error. Also, these deviations from sphericity happen (for reasonable black hole masses and neutron star densities) only when the neutron star is relatively close to the black hole, beyond which point the inertial frame used violates the assumptions used in its constructionnamely, that it be small. Therefore, one may consider that the behavior of the ellipsoid model gives only a rough idea of the velocities of the neutron star material. The only reasonably simple approximation is to treat this material as being "free" after some point in the infall is reached. By "free," it is meant that the material follows geodesics in the background Schwarzschild metric; that is, further acceleration due to the rest of the neutron star matter, hydrodynamics, magnetic fields, nuclear reactions, tidal forces, etc., are neglected. Although each of the neglected forces may be significant in itself, each works at cross purposes. On the one hand, the material tends to slow down (if initially it is being ejected) because of the gravitational field of the rest of the neutron star material behind it. This field, of order $m\left(a_{1} a_{2} a_{3}\right)^{-1 / 3}$, 
is generally smaller than the field due to the black hole, of order $M / R$, but certainly not negligible. On the other hand, the material, which had to be pushed by hydrodynamical boosts caused by the tidal forces in order to be initially outgoing, is still being forced outward by these same forces. Furthermore, upon being removed from the gravitational potential well of the neutron star, the matter is undergoing decompression and heating (see below), thereby increasing its rate of expansion. If the material is ejected with greater than the neutron star's escape velocity, there would not appear to be any problem with expecting that the hydrodynamic boosts will enable the initial velocities of the material to be maintained. However, one may question the validity of the models integrated far enough for this to occur.

An important factor which has been overlooked so far is the rotation of the neutron star relative to the Fermi frame. According to the model results, the neutron star is tidally locked in orbit; the star tends to become extended with one bulge pointing toward the black hole. This is to be expected, of course, on the basis of classical analyses. Therefore, relative to the Fermi (inertial) frame, the neutron star rotates with an angular velocity approximately equal to the orbital angular velocity, which for Schwarzschild circular orbits is $\Omega=\left(M R^{-3}\right)^{1 / 2}$, or, for $R=6 M, \Omega=$ $4 \times 10^{-7}\left(M_{\odot} / M\right) \mathrm{cm}^{-1}$. A neutron star of density $\rho \sim 10^{15} \mathrm{~g} \mathrm{~cm}^{-3}$ has a breakup velocity $\omega \sim(\pi \rho)^{1 / 2} \sim$ $5 \times 10^{-7} \mathrm{~cm}^{-1}$. Thus the neutron star is rotating with an appreciable fraction of its breakup velocitythereby lowering its self-gravitational potential. This may increase the validity of the approximation scheme discussed above.

One computes the mass fraction ejected by considering many points (typically more than 100) located throughout the neutron star. Using the transformation equation

$$
\begin{aligned}
X^{i}= & \left.X^{i}\right|_{0}+\left.e_{(j)}^{i}\right|_{0} X^{(j)} \\
& +\left.\left(\frac{\partial e^{i}{ }_{(j)}}{\partial X^{\mu}} e^{\mu}{ }_{(k)}\right)\right|_{0} X^{(k)} X^{(j)}+\cdots,
\end{aligned}
$$

where the notation $/ 0$ means "evaluated at the center of the Fermi frame," the positions in the Schwarzschild frame are computed. The velocities are obtained by differentiating equation (55). Then assuming that all forces except the attraction due to the black hole cancel each other, one may compute an orbit for each mass point. If the particle's energy at infinity per unit mass is greater than 1 , and if its angular momentum per unit mass is greater than that needed for capture by the black hole, the mass point is assumed to "escape."

\section{RESULTS}

The initial scenario to be considered is that of an undeformed neutron star located far from the black hole on a nearly circular slowly spiraling trajectory.
It is computationally impractical to choose the initial radius of the orbit, $R$, very large. On the other hand, if the integration of the equations of motion is started at too small an initial radius, dependence of the results on the initial conditions becomes large. It is reasonable to expect, at least for large $R$, that the tidal forces produce a small deformation on the neutron star which tends to be constantly oriented toward the black hole: the neutron star should be tidally locked in orbit. This has been verified by introducing a small viscosity (see the Appendix) and integrating the neutron star's equations of motion, using initial conditions appropriate to an undeformed neutron star for very large $R$. Thus, the equilibrium deformation of the neutron star as a function of $R$ and $\bar{\rho} M^{2}$, where $\bar{\rho}$ is the average density in the neutron star, can be determined from the equations of motion in the rest frame (see the Appendix) by setting all time derivatives in equations (A2)-(A6) to zero, putting $\Omega(=-d \theta / d \tau)$ equal to $d \phi / d \tau$ (eq. [19]) and the vorticity parameter $\Lambda=0$, and solving. The results are illustrated in Figure 2. (Note that for each value of the parameter $\bar{\rho} M^{2}$, there exists a radius below which no stable equilibrium figures are possible. Fishbone [1972] has determined the relation between this radius and $\bar{\rho} M^{2}$ in the case of exactly circularly orbiting bodies [see Fig. 1 of LS]. The calculation shown in Fig. 2 takes into account the nongeodesic nature of the orbit, as well as the rotation of the neutron star caused by the tidal locking to the black hole.)

One may therefore remove from the results any

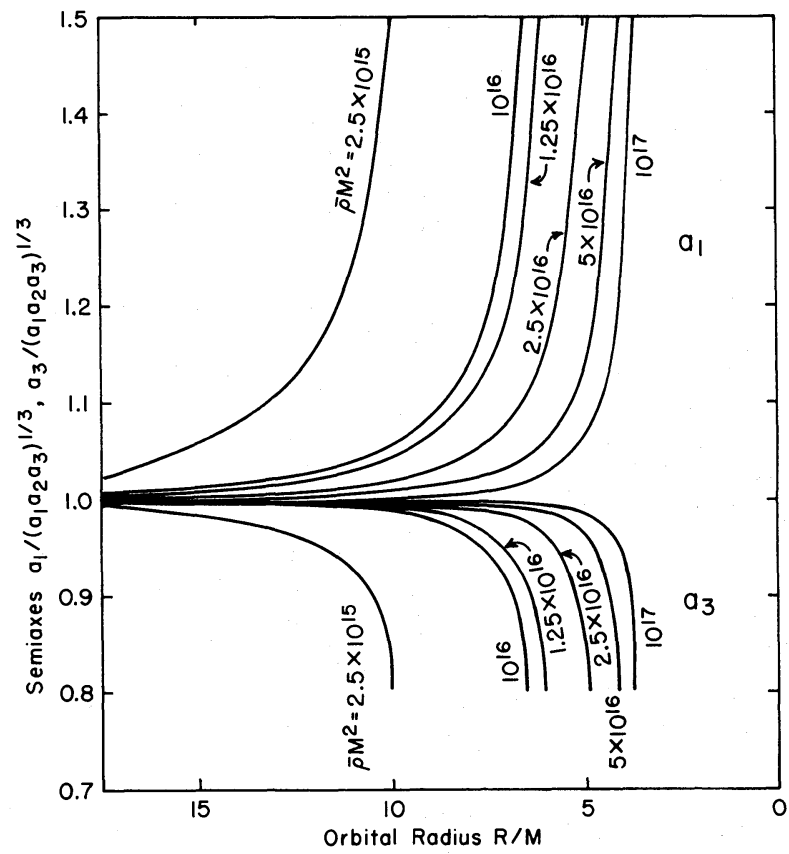

FIG. 2.-The equilibrium shapes of constant density $\bar{\rho}$ ellipsoids located a distance $R$ (in units of the black hole mass $M)$ from the black hole. The semiaxes $a_{1}$ and $a_{3}$ are in units of the neutron star radius at infinity, $\left(a_{1} a_{2} a_{3}\right)^{1 / 3}$. The ellipsoids have zero vorticity $\Lambda$ (see Appendix) and an angular velocity $\Omega$ appropriate to a tidally locked configuration (cf. eq. [16]). 


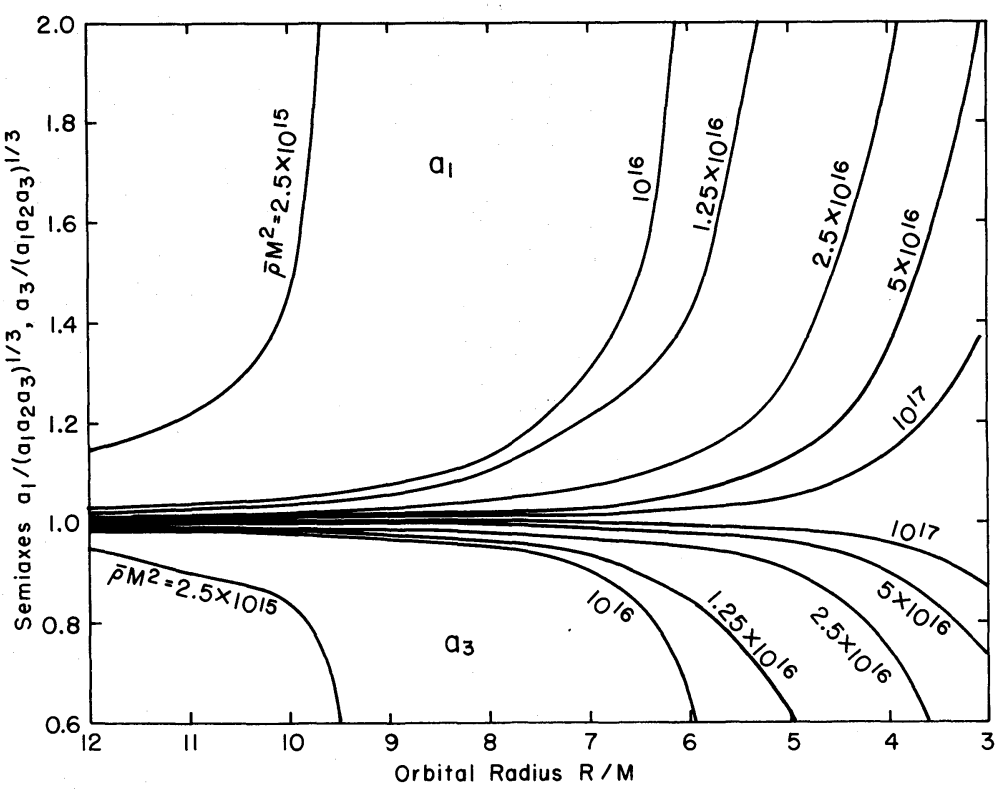

FIG. 3. - The semiaxes $a_{1}$ and $a_{3}$ of neutron stars of mean density $\bar{\rho}$ spiraling into black holes of mass $M$, according to the incompressible fluid model.

dependence on initial conditions, and still start the integration at a practical value of $R$, by choosing for the initial shape of the neutron star the equilibrium figure for the appropriate $R$ and $\bar{\rho} M^{2}$. Figure 3 shows the results of integrating the equations of motion (7), (19), (24), and (32) for an incompressible fluid ellipsoid. The axes $a_{1}$ (pointing toward the black hole) and $a_{3}$ are displayed for $1 M_{\odot}$ neutron stars of densities $\bar{\rho}=10^{14}$, $5 \times 10^{14}$, and $10^{15} \mathrm{~g} \mathrm{~cm}^{-3}$ falling into black holes of masses $M=5$ and $10 M_{\odot}$. It is found that the initial behavior of the neutron star is to follow closely the series of equilibrium figures shown in Figure 2 , bearing out the statement that the integrations are now free from dependence on initial conditions. As the tidal forces of the black hole grow, the deformations rapidly increase, and apparently result in the disruption of the neutron star.

Using the simple model described in \$ III for establishing if mass is ejected from the neutron star to infinity, the mass fraction ejected is plotted in Figure 4 as a function of the value of $R$ at which the ejection is assumed to take place. The onset of mass ejection is seen to be correlated with the rapid increase in the $a_{1}$ semiaxis. This is roughly where the velocity of expansion of $a_{1}$ exceeds the infalling orbital velocity of the center of mass of the neutron star. The expansion of the neutron star is seen to be monotonic. Mass ejection is predicted for $\bar{\rho} M^{2} \leqslant 10^{17} \mathrm{~g} M_{\odot}^{2} \mathrm{~cm}^{-3}$, at which point the models tend to become invalid.

The behavior of the ellipsoidal shell model discussed in $\S$ III is now examined. Three different equations of state have been employed, corresponding to a relatively soft, an intermediate, and a hard relationship, respectively. They are interpolated from the models B, D, and I of Arnett and Bowers (1974). In each case, the central density $\rho_{c}$ was chosen to give a neutron star mass $m$ near $1 M_{\odot}: \rho_{c, \mathrm{~B}}=2 \times 10^{15} \mathrm{~g} \mathrm{~cm}^{-3}$, $m_{\mathrm{B}}=0.97 M_{\odot} ; \rho_{c, \mathrm{D}}=10^{15} \mathrm{~g} \mathrm{~cm}^{-3}, m_{\mathrm{D}}=1.06 M_{\odot}$; $\rho_{c, \mathrm{I}}=5 \times 10^{14} \mathrm{~g} \mathrm{~cm}^{-3}, m_{\mathrm{I}}=1.2 M_{\odot}$; the subscripts refer to the Arnett and Bowers (1974) tabulated model. The equilibrium figures are found by setting $Q=d Q / d \tau=0$ in equation (24), and employing (29), (38), (49), (50), and (53) to determine the initial values of the $a_{i}$. The equations of motion (7), (19), (24), and (32) have been integrated as before, where now equations (49) and (50) are used in place of (40) and

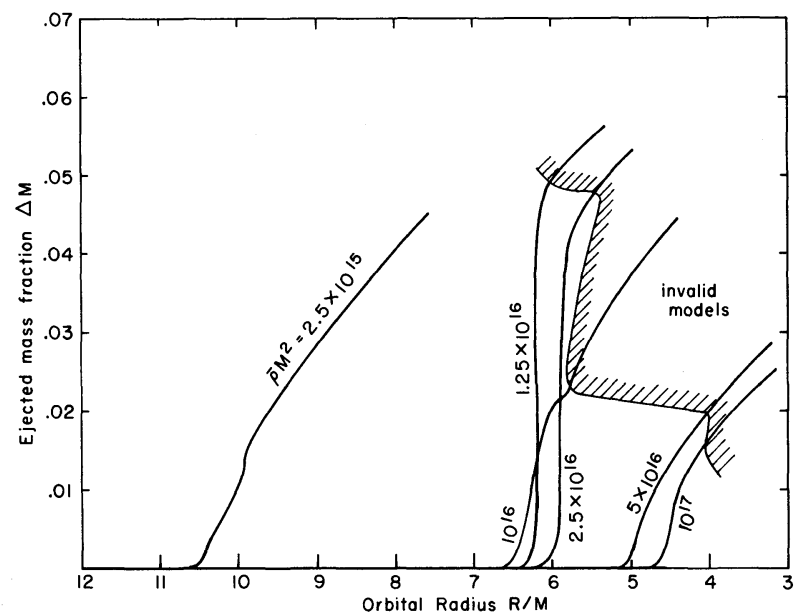

Fig. 4.-The mass fraction $\Delta M$ ejected for the models parametrized by $\bar{\rho} M^{2}$ illustrated in Fig. 3 . The ejected mass fraction is plotted against the value of the radial coordinate $R$ at which the neutron star matter is assumed to follow free particle trajectories. The region in which this model becomes invalid lies to the right of the dashed line, which corresponds to $\left(M / R^{3}\right)^{1 / 2} a_{1} \geq 0.2$, with $\left(a_{1} a_{2} a_{3}\right)^{1 / 3} \sim M$. 


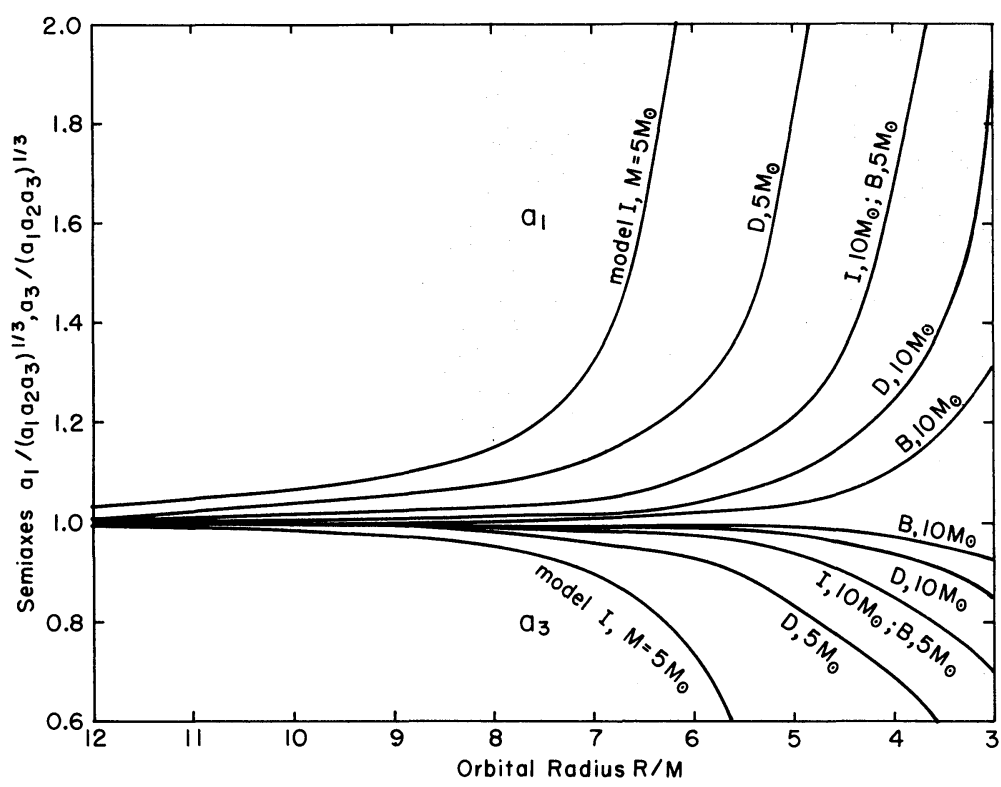

Fig. 5.- Same as Fig. 3, but for shelled ellipsoid models that fit the tabulated models B, D, and I of Arnett and Bowers (1974). The curves for cases $\left(\mathrm{I}, M=10 M_{\odot}\right)$ and $\left(\mathrm{B}, M=5 M_{\odot}\right)$ are nearly the same.

(39); instead of eliminating $\Pi$ from equation (24), one now employs equation (53). The infall is again considered for black hole masses of 5 and $10 M_{\odot}$.

Figure 5 illustrates the behavior of the axes $a_{1}$ and $a_{3}$ as the neutron star spirals into the black hole. Note that $\bar{\rho} M^{2}$ of each configuration is the determining factor in the location of the neutron star breakup radius. The precise form of the equation of state has been found to be unimportant in these results for models with equal densities. However, the equation of state has a major effect when comparing models of neutron stars containing equal mass. Current thinking favors stiff equations of state since the observed mass of the compact star (presumably a neutron star) in Her X-1 is $\sim 1.5 M_{\odot}$ (see, e.g., Milgrom and Salpeter 1975). Only stiff equations of state can account for such a large mass. However, since soft equations of state cannot yet be entirely ruled out, model B has been included to make our discussion more complete. The average density $\bar{\rho}$ of these configurations is generally about one-half of the corresponding central densities: $\quad \bar{\rho}_{\mathrm{B}}=1.2 \times 10^{15}, \quad \bar{\rho}_{\mathrm{D}}=6.7 \times 10^{14}$, and $\bar{\rho}_{\mathrm{I}}=3.1 \times 10^{14} \mathrm{~g} \mathrm{~cm}^{-3}$. It is important to note from Figures 2 and 5 that a shelled model with $\bar{\rho}=\rho_{0}$ behaves similarly to a constant density model with $\rho=\rho_{0}$.

The fraction of mass ejected is displayed in Figure 6 for each of these six cases. Generally, it is seen that the breakup point lies at a larger $R$ than in the constantdensity case for the same average density. Also, the mass fraction ejected is slightly smaller because there is less mass in the outer portions of the neutron star (the region that is ejected). Mass ejection is again seen to occur, within the limits of validity of these models, for $\bar{\rho} M^{2}<10^{17} \mathrm{~g} M_{\odot}{ }^{2} \mathrm{~cm}^{-3}$.

This brings one to a discussion of the validity of these models. When the quantities denoting the levels of the approximations used-namely, $(m / M)$ and $\left(M R^{-3}\right)^{1 / 2} r$, where $r$ is the extent of the neutron starexceed $0.1-0.2$, these models are probably invalid: their reliability as first guesses is questionable. Thus one may not consider cases for which $m / M>0.2$. Similarly, since the radius $r$ of a $1 M_{\odot}$ neutron star is approximately equal to $M$ for a $10 M_{\odot}$ black hole, the calculations become unreliable for $R<3 M$, if the extent of the neutron star is $r$. If, however, the neutron star is deformed to $2 r \sim 2 M$, then the calculations are unreliable for $R<4.6 M$. For a $5 M_{\odot}$ black hole, the calculations become unreliable for $R<$ $4.6 M$ or $7.4 M$ if the extent of the neutron star is $r \sim 2 M$ or $2 r \sim 4 M$, respectively.

The simplicity of the mass ejection model introduces more uncertainty into these calculations. For $\bar{\rho} M^{2} \leqslant$ $5 \times 10^{16} \mathrm{~g} M_{\odot}^{2} \mathrm{~cm}^{-3}$, the breakup of the neutron star is accompanied by surface velocities in the inertial frame characteristically greater than $0.1 c$. For $\bar{\rho} M^{2}>5 \times 10^{16} \mathrm{~g} \mathrm{M}_{\odot}^{2} \mathrm{~cm}^{-3}$, ejection velocities may not reach $0.1 c$ until the model has become invalid because the condition $\left(M R^{-3}\right)^{1 / 2} r \ll 1$ is violated. Escape velocities are near $0.1 c$ for a $1 M_{\odot}$ neutron star. Lower-density stars have greater deformations and larger radii; hence, the escape velocity also tends to be lower. The model used to describe mass ejection would seem more reasonable if escape velocities from the neutron star itself can be reached. This apparently happens, within the limits imposed by our approxi-

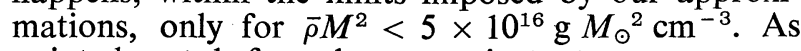
pointed out before, however, instantaneous escape velocities need not be reached if hydrodynamic (due to decompression and heating) and tidal forces accelerate the ejecta further. It is interesting to note here that this model for mass ejection does not require that the 


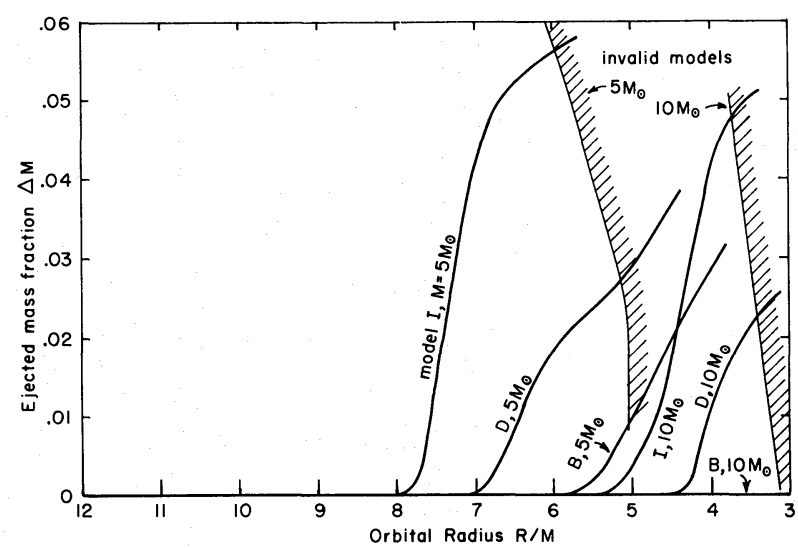

FIG. 6.-Same as Fig. 4, but for shelled ellipsoid models that fit the tabulated models B, D, and I of Arnett and Bowers (1974). The boundary of the invalid region is determined by $\left(M / R^{3}\right)^{1 / 2} a_{1}=0.2$, for the cases $M=5 M_{\odot}$ and $M=10 M_{\odot}$.

radial velocity $d R / d \tau$ with respect to the black hole be positive in order for free particles to escape to infinity. This is a result of the large angular velocities that the neutron star has since it is tidally locked in orbit. Matter near the surface along the $a_{1}$ axis pointing away from the black hole is boosted in the direction of the orbital motion, making escape from the black hole easier. Generally, though, the mass ejection is more prodigious when the material is ejected only along the $a_{1}$ axis sufficiently to overcome the net infalling motion of the neutron star.

It is possible that the low-density outer portions of the neutron star may be stripped off before the core is disrupted by the combination of tidal forces and the rotation of the neutron star; this behavior was noted for earlier two-component shelled models. However, the ejection velocity is probably too small for any of this material to escape the system. It is also possible that a continuous "stripping off" process could result in the neutron star losing an appreciable fraction of its mass; expanding, as a result, it could reach its "Roche limit" by lowering its density. It is not clear, however, that the loss of the outer layers could be achieved fast enough (i.e., on an orbital time scale of about $0.01 \mathrm{~s}$ ) for this to occur.

There is one last feature of the mass ejection worth mentioning. The ejected mass comes from a narrow cone around $a_{1}$, the semiaxis of the neutron star pointed away from the black hole. It is possible that a jet is forming; but it is impossible, without a more accurate picture of mass ejection, to verify this.

The models of massive stars of Arnett and Schramm (1973) indicate that stars more massive than $6 M_{\odot}$ tend to evolve into presupernova configurations of a collapsing $1.4 M_{\odot}$ core. However, about $0.1 M_{\odot}$ of the core is presumably ejected in a supernova (Hainebach, Norman, and Schramm 1976). Thus the collapsing neutron star presumably has a mass of $\sim 1.3 M_{\odot}$. Arnett and Bowers (1974) models B, D, and I give, for $1.3 M_{\odot}$ neutron stars, the average densities $\bar{\rho}_{\mathrm{B}, 1.3}=1.9 \times 10^{15}, \bar{\rho}_{\mathrm{D}, 1.3}=7.9 \times 10^{14}$ and $\bar{\rho}_{\mathrm{I}, 1.3}=$ $3.5 \times 10^{14} \mathrm{~g} \mathrm{~cm}^{-3}$. In the case of a $5 M_{\odot}$ black hole, all three values of $\bar{\rho} M^{2}$ are less than $5 \times 10^{16} \mathrm{~g} M_{\odot}{ }^{2}$ $\mathrm{cm}^{-3}$ : the models then imply that mass ejection is possible before they become invalid. However, the approximation $m / M \ll 1$ is becoming severely strained. For $M \sim 10 M_{\odot}$, mass ejection can be predicted only for models D and I; model B may also eject matter, but this model may not be extrapolated that far. Black hole masses larger than 7,11 , or $17 M_{\odot}$ make it impossible for the models to say anything significant about mass ejection for the equations of state $B, D$, or I, respectively (since, for these cases, $\bar{\rho} M^{2}>$ $10^{17} \mathrm{~g}_{\odot}^{2} \mathrm{~cm}^{-3}$ ). Therefore, it may be concluded that it is not implausible for some neutron star matter to be ejected in a black-hole-neutron-star collision for a wide range of black hole masses and neutron star equations of state.

These calculations may be generalized to include rotating black holes (Lattimer 1976). It is found that the equilibrium breakup points for incompressible fluids lie only slightly closer to the black hole than in the nonrotating hole case (Fig. 2), even for maximally rotating Kerr holes (see also Fishbone 1973). It seems, therefore, that the results of this paper may also apply to Kerr black holes. This problem is being further investigated.

\section{IMPLICATIONS AND DISCUSSION}

The discussion in $\S$ II illustrates that black-holeneutron-star pairs may not be rare. The fact that the X-ray star in the Cyg X-1 system is apparently more massive than $10 M_{\odot}$ suggests that black holes can be formed in close binaries. Section II also shows that most doubly compact binaries with one member a black hole can, in many cases, be formed with the members close enough to decay completely within the age of the Universe because of gravitational radiation. The observations and theoretical models seem to indicate that most neutron stars may be relatively massive $\left(\sim 1.3 M_{\odot}\right)$. Coupled with the inferred moments of inertia of observed pulsars (Arnett and Bowers 1974), only relatively stiff equations of state are allowed; it may be that model $B$ can even be ruled out. From the results presented in $\S$ IV, only models for which $\bar{\rho} M^{2}$ is less than $(5-10) \times 10^{16} \mathrm{~g} \mathrm{M}_{\odot}{ }^{2} \mathrm{~cm}^{-3}$ can be said to probably eject matter to infinity without the model becoming completely unreliable. One may not, however, rule out the possibilities that no mass is ejected from a neutron star of any density, or that mass is ejected from high-density neutron stars. If one uses an intermediate equation of state (say model D), then our models indicate that black holes less massive than about 8-11 $M_{\odot}$ would be capable of ejecting significant mass from a $1.3 M_{\odot}$ neutron star. For $\bar{\rho} M^{2}$ much greater than $2 \times 10^{17} \mathrm{~g} \mathrm{M}_{\odot}^{2} \mathrm{~cm}^{-3}$, the breakup of the neutron star will probably lie inside the Schwarzschild radius of the black hole. No ejected mass can be obtained in such a case.

Because of the inaccuracies of these models, and because both the mass and the proper equation of state of a neutron star in these systems are unclear, it is not possible at this time to accurately estimate 
the fraction of black-hole-neutron-star binaries that will eventually eject matter. An upper limit to the importance of these systems which is not inconsistent with these models can be obtained by setting this fraction equal to unity. If mass ejection occurs, these models show, in agreement with LS, that a reasonable upper limit for the fraction of the neutron star ejected is $\sim 5$ percent, so $\sim 0.05 M_{\odot}$ may be used as the amount of mass ejected per collision.

In $\S$ II we arrived at an estimate for the birthrate of possible progenitors of black-hole-neutron-star binaries, based on the numbers of massive X-ray binaries within $3 \mathrm{kpc}$. Note that this birthrate, $(2-5) \times 10^{-12} \mathrm{pc}^{-2} \mathrm{yr}^{-1}$, is consistent with the number of close binaries (separations less than a few AU) formed by stars greater than $\sim 15 M_{\odot}$. The birthrate of stars greater than $15 M_{\odot}$ is given in Ostriker, Richstone, and Thuan (1974) as $\sim 3 \times 10^{-12} \mathrm{pc}^{-2} \mathrm{yr}^{-1}$. The binary statistics of Heintz (1969) indicate that about one-third of all binaries have a smaller separation than $3 \mathrm{AU}$, and that there is at least one double star system per 4 total stars. These statistics hold in particular for massive stars. Therefore, the birthrate of binaries with a massive O-B star that may lead to massive X-ray binaries is $\sim 5 \times 10^{-13} \mathrm{pc}^{-2} \mathrm{yr}^{-1}$, in reasonable agreement with observations. The fraction of these binaries that form black-hole-neutron-star systems is difficult to estimate; but since one of the three systems within $3 \mathrm{kpc}$ may have a black hole in it, a fraction of about 10 percent may be reasonable. Since virtually all black-hole-neutron-star systems formed via these close binaries should eventually lead to neutron star breakups, one sees that the death rate of these binaries is $2 \times 10^{-13} \mathrm{pc}^{-2} \mathrm{yr}^{-1}$. Finally, since the rate of star formation $\Psi$ was higher in the past, a correction $\langle\Psi\rangle / \Psi_{p}$, where $\langle\Psi\rangle$ is the average of $\Psi$ over the age of the Galaxy and $\Psi_{p}$ is the present value of $\Psi$, should be applied to this number, along with the age of the Galaxy $\left(\sim 10^{10} \mathrm{yr}\right)$, to arrive at the total number of black-hole-neutron-star collisions that have occurred per square parsec in the plane of the galaxy. The correction $\langle\Psi\rangle / \Psi_{p}$ is, conservatively, 10 (see, e.g., Ostriker, Richstone, and Thuan 1974). If $0.05 M_{\odot}$ of former neutron star material is ejected in each collision, then one sees that $10^{-3} M_{\odot} \mathrm{pc}^{-2}$ of ejected neutron star material has enriched the interstellar medium. Oort (1963) gives the average density in the solar neighborhood as $75 M_{\odot} \mathrm{pc}^{-2}$, so this leads to a maximum ejected mass fraction of $1.3 \times 10^{-5}$. Comparing this number with the observed abundances of $r$-process nuclei, (1.5-1.8) $\times 10^{-6}$ (Burbidge et al. 1957), and deuterium, $1.2 \times 10^{-5}$ (Rogerson and York 1974), one sees that these events could have considerable influence upon the nucleosynthesis in the Galaxy.

What sort of nuclei might one expect to be formed in the breakup of a neutron star? To estimate an answer, consider the structure of a pre-breakup, ground-state neutron star. Basically, the mass of a neutron star lies in three regimes (Baym and Pethick 1975). The outer layers, comprising a negligibly small fraction (typically less than $0.01 \%$ ) of the total mass, lie at densities less than about $4 \times 10^{11} \mathrm{~g} \mathrm{~cm}^{-3}$ and can be ignored. The intermediate region, composed of neutron-rich nuclei immersed in a sea of free ("dripped") neutrons, is at densities between $4 \times 10^{11}$ and $3 \times 10^{14} \mathrm{~g} \mathrm{~cm}^{-3}$ (nuclear density). The third region is the center of the neutron star, and is composed mainly of neutrons, hyperons, and a few percent of protons and electrons. During the breakup, the density decreases on a (free-fall) time scale $\sim 446 \rho^{-1 / 2} \mathrm{~s}$. Because nuclear time scales are so much shorter, matter at densities greater than nuclear densities expands in equilibrium; when the density falls below nuclear densities, the matter quickly achieves a structure similar to equilibrium matter of densities just below $3 \times 10^{14} \mathrm{~g} \mathrm{~cm}^{-3}$. Thus one need only consider the nonequilibrium behavior of matter in the intermediate region.

The nuclei in this region, being surrounded by a free neutron gas, are extremely neutron-rich. As the matter expands, there is a tendency for these nuclei to become smaller, that is, for neutrons to be pulled out of the nuclei. Also, the matter will cease to be in $\beta$-equilibrium, since

$$
\Delta=E_{\mathrm{F} e}-E_{\mathrm{F} n}+E_{\mathrm{F} p}
$$

becomes nonzero, where $E_{\mathrm{Fe}}, E_{\mathrm{F} n}$, and $E_{\mathrm{F} p}$ are the Fermi energies of the neutrons, protons, and electrons, respectively. $\Delta$ is the maximum energy available to the antineutrino. To estimate how the $\beta$-decay rate depends on $\Delta$, one may treat the decay like the decay of an "ordinary" nucleus. Then the mean lifetime $\tau_{\beta}$ is given by (assuming the electrons to be degenerate)

$$
\frac{1}{\tau_{\beta}} \approx \frac{d}{f t}\left(\frac{5}{2} E_{\mathrm{Fe}}^{2} \Delta^{4}+E_{\mathrm{Fe}} \Delta^{5}+\frac{1}{6} \Delta^{6}\right),
$$

where $d$ is the density of states in the daughter nucleus and the " $f t$ "-value for allowed $\beta$-decays is in the range $10^{4}-10^{6} \mathrm{~s}-\mathrm{MeV}^{5}$.

Calculations (Lattimer et al. 1975) are in progress to analyze the expanding nuclear matter. During the initial stages of the expansion, no $\beta$-decays occur because the time scale involved is long compared with the expansion time scale, since $E_{\mathrm{Fe}} \sim 100 \mathrm{MeV}$, $d \sim 10 \mathrm{MeV}^{-1}$, and $\Delta \sim$ a few $\mathrm{MeV}$. Preliminary results suggest that when $\rho \sim 10^{11} \mathrm{~g} \mathrm{~cm}^{-3}, \Delta \sim$ $10 \mathrm{MeV}$ and $E_{\mathrm{Fe}} \sim 20 \mathrm{MeV}$, so that the two time scales are comparable and $\beta$-decays begin. The mass of the nucleus now begins to increase; the increase in the proton number causes the neutron number to increase also. As a result the nuclei now appear to follow a path parallel to the so-called $r$-process path in the $Z-N$ plane toward the region of superheavy elements. This path, however, lies somewhat to the neutron-rich side of the $r$-process path. Eventually, the path of these nuclei enters into regimes unstable to fission. An average $\beta$-decay releases the energy $\Delta \sim 10 \mathrm{MeV}$. Fissions release even more energy. Thus the matter can no longer be cold; temperatures near $10^{10} \mathrm{~K}$ are reached. This is high enough to cause the matter to approach nuclear statistical equilibrium. There are still large numbers of free neutrons outside 
the nuclei; these are rapidly absorbed as the expansion continues. An $r$-process-like cycling of the nuclei is set up. At present, it is unclear whether an $r$-processlike distribution of heavy elements results, although it seems safe to conclude that neutron-rich heavy elements themselves do result.

Because of the extremely fast neutron capture rates of these nuclei one can show that essentially no free neutrons can be ejected in the breakup of a neutron star. Assume that the neutron-capture cross section of the nuclei $\sigma$ times the average neutron thermal velocity $V_{n}$ is $\sim 1 \mathrm{mb} \times 10^{7} \mathrm{~cm} \mathrm{~s}^{-1}$, a conservative estimate (Blake and Schramm 1973). The lifetime $\tau_{n}$ of a nucleus against capturing a neutron is

$$
1 / \tau_{n} \approx n \sigma V_{n}
$$

where $n$ is the free-neutron number density. From the models of Lattimer et al. (1975), the free-neutron density vanishes when the mass density of the nuclei is near $10^{11} \mathrm{~g} \mathrm{~cm}^{-3}$. Assume that at this density there is one free neutron per nucleus (average mass $A \sim 300$ ). Thus $n=10^{11} \mathrm{~g} \mathrm{~cm}^{-3} \times N_{\mathrm{A}} / 300 \sim 2 \times 10^{32} \mathrm{~cm}^{-3}$, where $N_{\mathrm{A}}=$ Avogadro's number. As a result, $\tau_{n} \sim$ $5 \times 10^{-14} \mathrm{~s}$, about 10 orders of magnitude faster than the expansion time scale. Because the neutron capture rate is tied to the expansion rate, no free neutrons can escape. Since virtually no free neutrons survive, one may rule out the formation of deuterium by this mechanism.

That a large number of $\beta$-decays occur in the breakup of a neutron star is clear. An antineutrino is emitted for each such $\beta$-decay. However, the $\bar{\nu}$ spectrum emitted by a black-hole-neutron-star collision does not seem to agree with the recently observed antineutrino burst (Lande et al. 1974). First of all, the energies available to the antineutrinos on the basis of the above picture are about $10 \mathrm{MeV}$ or so, while energies in the range 20-100 MeV were observed. Secondly, causality would prohibit a source of the size $\sim 10^{6} \mathrm{~cm}$ from producing a pulse width $\sim 1 \mu \mathrm{s}$ as observed, unless the source were extremely relativistic (see Bludman and Ruderman 1975). This is not in accordance with our model. Since the time scale of expansion of the neutron star is about a factor of $10^{3}$ greater than $1 \mu \mathrm{s}$, the expansion velocity is about $0.1 c$, i.e., nonrelativistic. Third, the observed intensity of the event is too large to have been produced by weak interactions in a nonrelativistic source of the mass of a neutron star (Bludman and Ruderman 1975). This is easily seen from the relation

$$
F \tau=\frac{L \tau}{4 \pi r^{2}} \leqslant \frac{\tau}{4 \pi r^{2}} \frac{N \Delta}{\tau_{\beta}},
$$

where $\tau$ is the pulse time ( $\left.\sim \frac{1}{2} \mu s\right), F$ the flux observed, $L$ the luminosity, $r$ the distance to the source, and $N$ the number of baryons able to undergo $\beta$-decay. $F \tau$ was observed to be $2 \times 10^{7} \mathrm{ergs} \mathrm{cm}^{-2}$ (Lande $e t$ al. 1974); $N \sim 6 \times 10^{56}, \tau_{\beta} \sim 10^{-3} \mathrm{~s}$, and $\Delta \sim 10 \mathrm{MeV}$ from the above discussion. Therefore, $r \sim 5 \times 10^{19} \mathrm{~cm}<$ $17 \mathrm{pc}$. The source, if extraterrestrial, must have been more distant because of the impossibility of masking the optical, X-, and $\gamma$-ray photons that would certainly be emitted. It would therefore seem that the antineutrino burst of Lande et al. (1974) was probably not created in this manner.

However, as neutrino detection apparatus begins to probe lower energies, it may be possible to observe an antineutrino burst from a black-hole-neutron-star collision. The frequency of these events will be prohibitively low unless much smaller burst intensities can be recorded. A source $3 \mathrm{kpc}$ distant, for example, would be expected to give rise to a burst of duration $\tau \sim 1$ ms containing the energy $(F \tau)$ given by equation (59), or $\sim 8 \times 10^{6}$ ergs $\mathrm{cm}^{-2}$. Even if all the massive $X$-ray binaries within $3 \mathrm{kpc}$ give rise to such an event, their frequency would be about $10^{-4} \mathrm{yr}^{-1}$, or practically unobservable. To have any chance of being observed in this manner, therefore, all of the sources within the Galaxy would have to be observable and the required sensitivity of an antineutrino detector would have to be $\sim 10^{5}$ ergs $\mathrm{cm}^{-2}$ near energies of $10 \mathrm{MeV}$.

Whether or not mass ejection takes place in these events, prodigious amounts of electromagnetic radiation are undoubtedly emitted. This radiation will occur either from the breakup of the neutron star itself, or the subsequent accretion of some of the dispersed material. Again, to be observable with a reasonable frequency, all black-hole-neutron-star collisions occurring within the Galaxy must be visible. The maximum frequency of these events is then $0.01 \mathrm{yr}^{-1}$, which is far too low to explain $\gamma$-ray bursts. Because of the low frequency of these events, their most important observational consequence may be nucleosynthesis.

In the Introduction, it was suggested that double neutron star binaries may exist. The binary pulsar may be an example of such a system; furthermore, equation (3) predicts that the components should collide within the age of the Universe. The ellipsoidal models of $\S$ III cannot be applied to these systems since $m / M \sim 1$. One may speculate, however, that some neutron star matter might be ejected, providing an additional source of neutron-rich nuclei. Nevertheless, to order of magnitude, the statistical arguments of this section are not affected.

\section{SUMMARY}

Massive X-ray binaries may evolve to systems containing two compact stars. The probability that these systems remain bound is enhanced if at least one of the compact stars is a black hole. Since Cyg X-1 appears to contain a black hole (presumably as a result of the supernova process), the formation of black holes in binaries may be commonplace. The binary pulsar may be evidence that doubly compact binaries exist and that two supernovae may occur without the disruption of the binary.

If black-hole-neutron-star binaries are formed, they are likely to have separations small enough to result in a collision due to the emission of gravitational radiation. The tidal forces of the black hole cause the disruption of the neutron star before the neutron star 
falls into the black hole for configurations which satisfy $\bar{\rho} M^{2} \leqslant(5-10) \times 10^{16} \mathrm{~g} \mathrm{M}_{\odot}^{2} \mathrm{~cm}^{-3}$. On the basis of a simple model, the disruption is violent enough to cause the ejection of a significant fraction ( $\leqslant 5$ percent) of the neutron star. Note that this upper limit, for a $1.3 M_{\odot}$ neutron star (suggested by evolutionary calculations), corresponds to maximum black hole masses in the range 8-17 $M_{\odot}$, depending on the neutron star equation of state. The approximations used in this model break down for higher values of $\bar{\rho} M^{2}$ before mass is observed to be ejected.

Although the frequency of these events is too small to be important as far as currently observed antineutrino, $\gamma$-ray, or optical bursts are concerned, enough neutron star material may be ejected to be of nucleosynthetic importance. Rough estimates of the composition of the ejected nuclei indicate that heavy neutron-rich nuclei are produced, including, perhaps, the almost mythical superheavy elements. However, it seems almost impossible that any significant amount of deuterium is formed. Nevertheless, black-holeneutron-star interactions may occur frequently enough to account for some of the presently observed $r$-process material.

We wish to acknowledge valuable discussions with W. D. Arnett, G. Baym, D. Gunter, J. Ipser, F. Mackie, G. Ravenhall, and J. C. Wheeler. J. M. L. was supported by the Benfield Fellowship of The University of Texas at Austin during the period when most of this research was performed, and wishes to acknowledge the hospitality of the Enrico Fermi Institute of the University of Chicago where part of this research was performed. This research was supported in part by NSF grant AST 74-21216 at the University of Chicago.

\section{APPENDIX}

In the classical problem of Dirichlet, a Newtonian, self-gravitating, perfect fluid of constant density is constrained to an ellipsoidal shape. Rotating ellipsoids having two equal semiaxes perpendicular to the rotation axis are known as Maclaurin spheroids. See, for example, Chandrasekhar (1969) for a thorough discussion of classical ellipsoids. Rossner (1967) has investigated the evolution of Maclaurin spheroids with finite initial deformations, but has not considered the perturbing effects of viscosity, tidal forces, etc. Some investigators have examined the behavior of ellipsoids including these effects, but have concentrated on other cases such as evolution from the unstable portion of the Maclaurin sequence to the Riemann sequence. For example, Press and Teukolsky (1973) deal with viscosity, while Miller (1974) studies the effects of gravitational radiation reaction. Ellipsoids oscillating about the Maclaurin sequence pass back and forth through a point at which two moments of inertia are degenerate. This degeneracy causes the orientation of the rest frame of the ellipsoid with respect to an inertial frame to become ambiguous. It is shown below that this ambiguity is inherent in the equations describing the evolution of the ellipsoid with respect to its rest frame-in the form of a coordinate singularity. This singularity is simply eliminated (see below) if no perturbing influences such as viscosity and tidal forces are present. If, however, additional forces are considered, this singularity remains in the equations, apparently causing difficulty in their solution. The purpose of this Appendix is to demonstrate that by rewriting the equations with respect to an inertial frame the coordinate singularity does not appear. A consistent picture of the oscillations about the perturbed Maclaurin sequence can thus be obtained. Moreover, analysis of the solutions obtained with the inertial frame method gives the result that the solutions obtained from the original (rest frame) equations are fully compatible, despite the apparent singularity.

Chandrasekhar (1969) develops the rest frame equations for the ellipsoid's evolution, including viscous dissipation. Miller (1974) extends these equations to include gravitational radiation reaction, while Mashhoon (1972) adds a tidal force term. Consider the effects of including viscous dissipation and tidal forces. Assume that the tidal force is derivable from a potential $\Phi$ (eq. [35]), so that

$$
R^{\prime}{ }_{i j}=R_{(i)(0)(k)(0)}^{\prime} I^{\prime}{ }_{k j} .
$$

$R_{(i)(0)(j)(0)}^{\prime}$ is symmetric and traceless; primes denote rest frame values as before. Also assume without loss of generality that the angular velocity $\Omega$ and vorticity of the ellipsoid lie only in the " 2 " direction. Then the only nonzero off-diagonal elements of $R_{i j}^{\prime}$ are $R_{13}^{\prime}$ and $R_{31}^{\prime}$. One may then write the evolution equations for a constantdensity, incompressible fluid ellipsoid as

$$
\begin{aligned}
& \frac{d^{2} a_{1}}{d t^{2}}=a_{1}\left(\Omega^{2}+\Lambda^{2}\right)-2 a_{3} \Omega \Lambda+\frac{B_{11}^{\prime}}{a_{1}}+\frac{\Pi}{a_{1}}-\frac{R_{11}^{\prime}}{a_{1}}-\frac{2 \nu_{0}}{a_{1}{ }^{2}} \frac{d a_{1}}{d t}, \\
& \frac{d^{2} a_{2}}{d t^{2}}=\frac{B_{22}^{\prime}}{a_{2}}+\frac{\Pi}{a_{2}}-\frac{R_{22}^{\prime}}{a_{2}}-\frac{2 \nu_{0}}{a_{2}{ }^{2}} \frac{d a_{2}}{d t}, \\
& \frac{d^{2} a_{3}}{d t^{2}}=a_{3}\left(\Omega^{2}+\Lambda^{2}\right)-2 a_{1} \Omega \Lambda+\frac{B_{33}^{\prime}}{a_{3}}+\frac{\Pi}{a_{3}}-\frac{R_{33}^{\prime}}{a_{3}}-\frac{2 \nu_{0}}{a_{3}{ }^{2}} \frac{d a_{3}}{d t}, \\
& \frac{d \Omega}{d t}=\frac{2}{\left(a_{1}^{2}-a_{3}^{2}\right)}\left[\frac{d a_{3}}{d t}\left(a_{3} \Omega+a_{1} \Lambda\right)-\frac{d a_{1}}{d t}\left(a_{1} \Omega+a_{3} \Lambda\right)+\frac{1}{2}\left(R_{31}^{\prime}+R_{13}^{\prime}\right)+\Lambda \nu_{0}\left(\frac{a_{3}}{a_{1}}-\frac{a_{1}}{a_{3}}\right)\right],
\end{aligned}
$$




$$
\begin{aligned}
& \frac{d \Lambda}{d t}=\frac{2}{\left(a_{1}{ }^{2}-a_{3}{ }^{2}\right)}\left[\frac{d a_{3}}{d t}\left(a_{1} \Psi+a_{3} \Lambda\right)-\frac{d a_{1}}{d t}\left(a_{3} \Omega+a_{1} \Lambda\right)+\frac{1}{2}\left(\frac{a_{1} R_{13}^{\prime}}{a_{3}}+\frac{a_{3} R_{31}^{\prime}}{a_{1}}\right)+\frac{\Lambda \nu_{0}}{2}\left(\frac{a_{3}{ }^{2}}{a_{1}{ }^{2}}-\frac{a_{1}{ }^{2}}{a_{3}{ }^{2}}\right)\right], \\
& a_{1} a_{2} a_{3}=\text { const. }
\end{aligned}
$$

The notation follows Chandrasekhar (1969). The $a_{i}(t)$ are the ellipsoid's semiaxes, and $\Lambda(t)$ is proportional to the vorticity. $\Pi(t)$ is related to the pressure $P$ by equation (26), and the $B^{\prime}{ }_{i j}$ are the rest frame versions of equation (27) (see eq. [39]). The quantity $\nu_{0}$ is an effective coefficient of kinematic viscosity; see, for example, Press and Teukolsky (1973).

Equations (A5) and (A6) become singular when $a_{1}=a_{3}$ : this is the point at which the two moments of inertia perpendicular to the angular velocity are equal. This degeneracy renders ambiguous the orientation of the ellipsoid with respect to an inertial frame. In this way the problem bears an analogy to the three-body problem (cf. Hill 1974).

Consider, first, the tide- and viscosity-free equations $\left(R_{i j}^{\prime}=\nu_{0} \doteq 0\right)$. The angular momentum $L$ and the circulation $C$ are defined by (Chandrasekhar 1969)

and

$$
\begin{aligned}
& L=I_{0}\left[\Omega\left(a_{1}{ }^{2}+a_{3}^{2}\right)-2 a_{1} a_{3} \Lambda\right] \\
& C=\pi\left[\Lambda\left({a_{1}}^{2}+{a_{3}}^{2}\right)-2 a_{1} a_{3} \Omega\right] .
\end{aligned}
$$

In the absence of viscosity, $C$ is conserved; while if there are no tidal forces (or gravitational radiation reaction), $L$ is conserved. It is then easy to show from equations (A5) and (A6) that

$$
\frac{d L}{d t}=\frac{d C}{d t}=0
$$

If the ellipsoid passes through the configurations $a_{1}=a_{3}$, equations (A8) and (A9) imply that $L=-C I_{0} / \pi$. Equation (A10) then shows that when $a_{1} \neq a_{3}, \Omega=-\Lambda$. Substituting this into equations (A5) and (A6) gives

or simply, by (A8),

$$
\frac{d \Omega}{d t}=-\frac{d \Lambda}{d t}=-\frac{2 L\left(d a_{1} / d t+d a_{3} / d t\right)}{I_{0}\left(a_{1}+a_{3}\right)^{3}},
$$

$$
\Omega=\frac{L}{I_{0}\left(a_{1}+a_{3}\right)^{2}} .
$$

Thus the singularity is easily removed in the case where there are no tidal or viscous forces; it is necessary to view the ellipsoid in a frame that rotates in such a manner as to have the vorticity contribute as much to the particle motion as the angular velocity (as viewed in an inertial frame). This simply corresponds to treating the rest frame of the ellipsoid as a frame rotating with $\Omega=-\Lambda=\Omega_{0} / 2$. Therefore, the angular velocity of the particles comprising the ellipsoid as viewed in an inertial frame is $\Omega_{0}=\Omega-\Lambda$.

The singularity is not easily removable when tidal forces are introduced. Returning to equations (A5) and (A6), and now setting $R_{i j}^{\prime} \neq 0$, one finds the angular momentum to be no longer conserved:

$$
\frac{d L}{d t}=I_{0}\left(R^{\prime}{ }_{31}-R_{13}^{\prime}\right)
$$

if ${R^{\prime}}_{(i)(0)(j)(0)}$ is a traceless, symmetric matrix. Thus, in general $L \neq-C I_{0} / \pi$; and unless $R^{\prime}{ }_{i j}$ vanishes when $a_{1}=a_{3}$, $d \Omega / d t$ and $d \Lambda / d t$ apparently become infinite. It would seem impossible, therefore, to be able to integrate the evolution equations through the point $a_{1}=a_{3}$. In fact, this effect can be seen in the work of Mashhoon (1972). His Figure IV.15 illustrates the point that equation (A5) implies-namely, that the angular velocity becomes so great as $a_{1}$ approaches $a_{3}$ that it becomes impossible for $a_{1}$ to become less than $a_{3}$. Rather, $a_{1}$ appears to "bounce."

The evolution of an initially slightly deformed ellipsoid far from the perturbing mass (so that the tidal force components $R_{(i)(0)(j)(0)}$ act as a small constant perturbation in the inertial frame) has been computed from equations (A2)-(A7) and is shown in Figure 7, as an example of the effects just discussed. The initial conditions for this computation are $a_{1}=1.01\left(a_{1} a_{2} a_{3}\right)^{1 / 3}, \quad a_{3}=0.99\left(a_{1} a_{2} a_{3}\right)^{1 / 3}, \quad d a_{i} / d t=R_{13}=R_{31}=0, \Omega=-\Lambda=-0.01$, and $R_{(1)(0)(1)(0)}=-2 R_{(2)(0)(2)(0)}=-2 R_{(3)(0)(3)(0)}=-2 \times 10^{-6}$ in the inertial frame (see eq. [29] to find the rest frame components of $\left.R_{(i)(0)(j)(0)}^{\prime}\right)$. Notice that even though $\Omega$ appears to become very large when $a_{1}$ approaches $a_{3}$, $\Omega-\Lambda$ remains nearly constant. It would appear from Figure 7 that $a_{1}$ and $a_{3}$ effectively change places at the singular point, which seems extremely nonphysical. In fact, eventually the computer calculation will become "stalled" when a value of $a_{1}-a_{3}$ is computed small enough. It is difficult, therefore, to follow the evolution of the ellipsoid in this frame. 


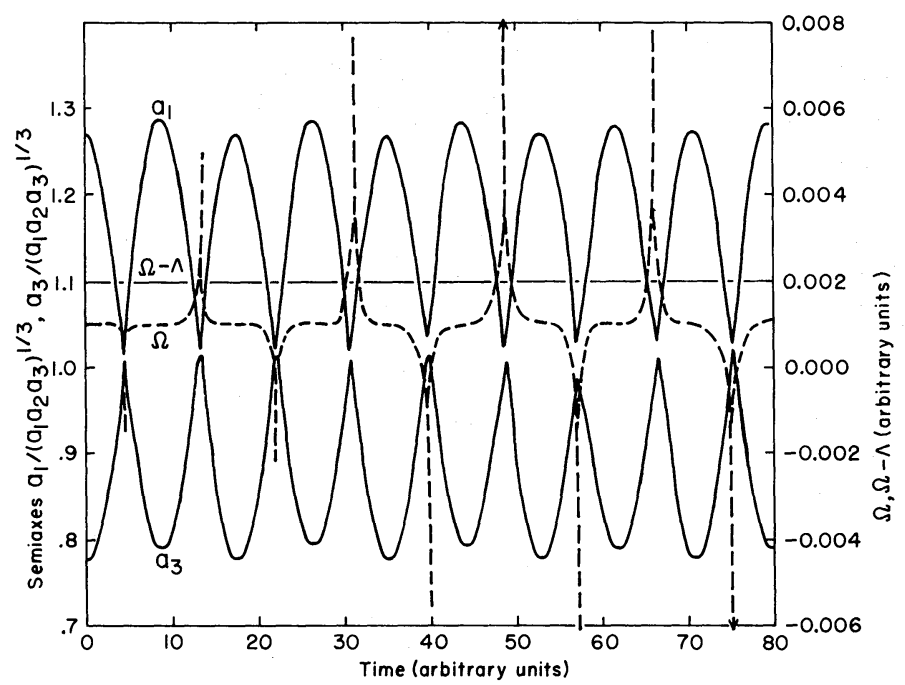

FIG. 7. - The evolution of the semiaxes $a_{1}$ and $a_{3}$, the angular velocity $\Omega$, and the vorticity parameter $\Lambda$ of a constant density incompressible ellipsoid in a constant small tidal field. These are the results of integrating the rest frame ellipsoid equations.

Since for a near-Maclaurin configuration, $\Omega-\Lambda$ gives the angular velocity of the constituent particles in the ellipsoid as viewed in an inertial frame, the constancy of $\Omega-\Lambda$ indicates that viewing this process in the inertial frame can shed some light on this problem.

Equations (21)-(32) in § III outline the development of the inertial frame equations. The effects of viscosity may be included, following Chandrasekhar (1969), by adding to the right-hand side of equation (21) the term

$$
\left.\frac{\partial}{\partial X_{k}}\left[\rho \nu\left\{\frac{\partial\left[d X_{i} / d t\right]}{\partial X_{k}} \frac{\partial\left[d X_{k} / d t\right]}{\partial X_{i}}-\frac{2}{3} \frac{\partial\left[d X_{l} / d t\right]}{\partial X_{l}} \delta_{i k}\right\}\right]=\frac{\partial}{\partial X_{k}} \llbracket \rho \nu\left\{Q_{i k}+Q_{k i}-\frac{2}{3} Q_{l l} \delta_{i k}\right\}\right],
$$

where $\nu$ is the coefficient of kinematic viscosity. This becomes an added term on the right-hand side of equation (24):

$$
-\int \rho v d V\left(Q_{i j}+Q_{j i}-\frac{2}{3} Q_{l l} \delta_{i j}\right) \equiv-m \nu_{0}\left(Q_{i j}+Q_{j i}-\frac{2}{3} Q_{l l} \delta_{i j}\right) .
$$

For an incompressible fluid, equations (39), (40) and

$$
I_{22}=\left(a_{1} a_{2} a_{3}\right)^{2}\left(I_{11} I_{33}-I_{13}^{2}\right)^{-1} ; \quad Q_{11}+Q_{22}+Q_{33}=0
$$

are valid.

One point deserves a comment. Equation (31), defining $\theta$, the angle through which the rest frame has rotated with respect to the inertial frame, gives a principal value for $\theta$ between $-\frac{1}{4} \pi$ and $\frac{1}{4} \pi$. As $I_{11}-I_{33}$ changes sign, $\theta$ will change abruptly by $\frac{1}{2} \pi$. Imposing the requirement that $\theta$ remain continuous means that whenever $I_{11}-I_{33}$ changes sign, $\frac{1}{2} \pi$ should be added to the value of $\theta$ as determined by equation (31). That this constraint does not affect the evolution of $I_{i j}$ is clear from equation (29), as $B_{i j}$ is unchanged.

The evolution of an initially slightly deformed ellipsoid in a small constant tidal field has again been computed. Because the ellipsoid is incompressible, $I_{22}$ and $Q_{22}$ may be eliminated by equation (A16). Assuming that $\nu_{0}=0$, equation (34) enables one to eliminate $Q_{31}$ since it is easy to show that

$$
\frac{d C}{d t}=\pi a_{1} a_{3}\left\{\frac{d}{d t}\left(Q_{31}-Q_{13}\right)+\left(Q_{11}+Q_{33}\right)\left(Q_{31}-Q_{13}\right)\right\}=0 .
$$

The appropriate initial conditions are now $I_{11}=a_{1}{ }^{2}, I_{33}=a_{3}{ }^{2}, Q_{i i}=0, Q_{13}=\Omega\left(a_{1}+a_{3}\right) / a_{3}, Q_{31}=$ $-\Omega\left(a_{1}+a_{3}\right) / a_{1} ; a_{1}, a_{3}, \Omega$, and $R_{i j}$ have the same values used in the rest frame computation. Equations (24) and (32) have been integrated to compute the remaining $(1,1),(3,3)$, and $(1,3)$ components of $I$ and $Q$.

As is expected, $I_{11}$ and $I_{33}$ undergo simple oscillations, shown in Figure 8. Note, though, that $a_{1}$ and $a_{2}$, determined from the $I_{i j}$ by equation (29), behave exactly in the manner found from the rest frame calculations. The quantity $a_{1}$, if initially larger than $a_{3}$, never becomes smaller than $a_{3}$; furthermore, $a_{1}$ and $a_{3}$ appear to "bounce" off each other at the point of closest approach.

This "bounce," or apparent interchange in the identities between $a_{1}$ and $a_{3}$, is seen to occur because of our demand that $\theta$ be continuous at these transition points. This is a necessary assumption, as Figure 9 shows. The evolution of $d^{2} a_{1} / d t^{2}$ and $d^{2} a_{3} / d t^{2}$, the acceleration of the surface of the ellipsoid along the " 1 " and " 3 " axes, 


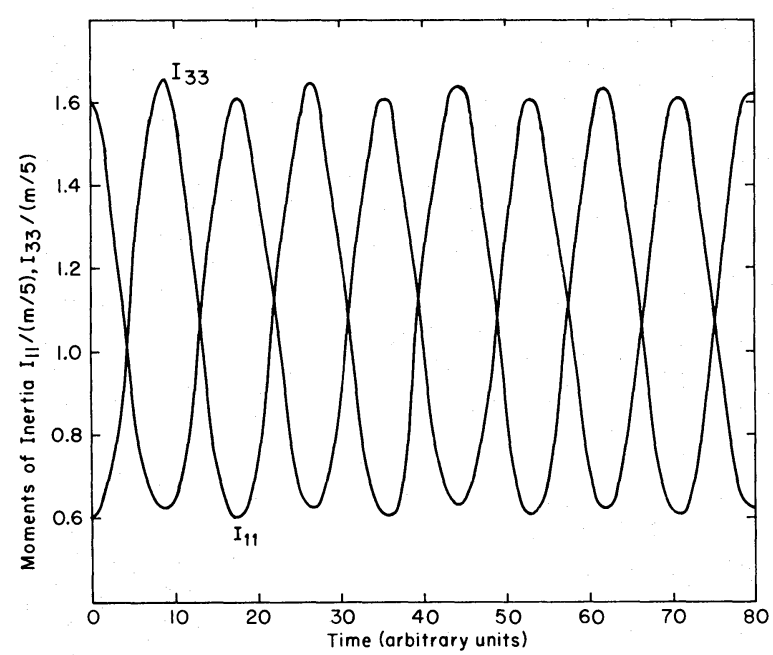

FIG. 8.- The evolution of the moments of inertia $I_{11}$ and $I_{33}$ in the inertial frame, as a result of integrating the inertial frame ellipsoid equations.

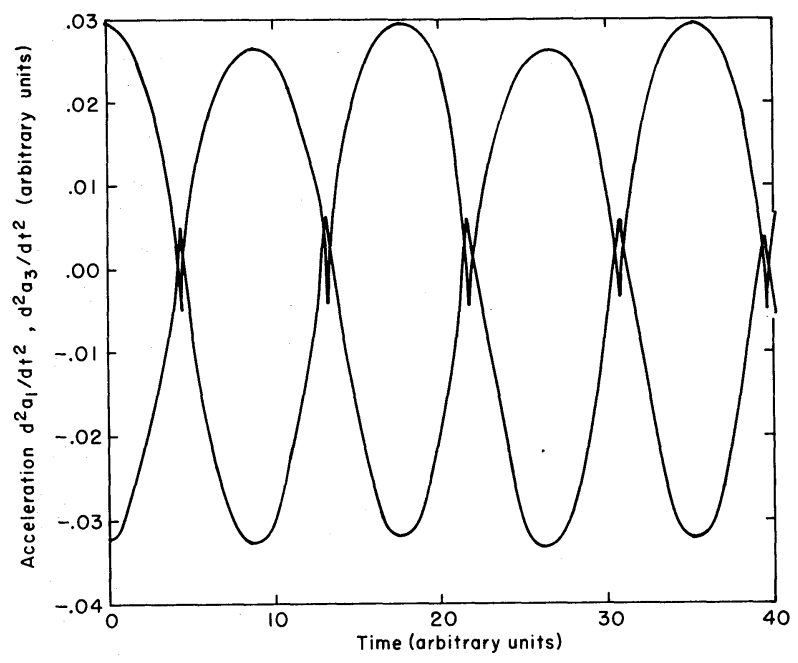

FIG. 9.-The rest frame accelerations $d^{2} a_{1} / d t^{2}$ and $d^{2} a_{3} / d t^{2}$ at the surface of the ellipsoid along the $a_{1}$ and $a_{3}$ axes, respectively, for the computations shown in Fig. A2.

respectively, is seen to be continuous at the points of closest approach of $a_{1}$ and $a_{3}$. These clearly would not be continuous if the requirement that $\theta$ be continuous were dropped. The fact that $\Omega$ rises to such large values is to ensure that $\theta$ change by $\pi / 2$ at the transition points. The angular velocity of the constituent particles in the ellipsoid is $\Omega-\Lambda$, which remains nearly constant during this change as demonstrated in the rest frame calculation.

The singularity in the rest frame calculation appeared in the equations for $d \Omega / d t$ and $d \Lambda / d t$ (eqs. [A5] and [A6]). By transforming to the inertial frame, $\Omega$ and $\Lambda$ can be found in terms of the tensors $I_{i j}$ and $Q_{i j}$. The solution of equations (24) and (32) for $d Q_{i j} / d t$ and $d I_{i j} / d t$ do not involve any singularities. Only by viewing the ellipsoid in the inertial frame can one arrive at a consistent view. Furthermore, the computer calculation proceeds much faster, and never becomes hung up at a singularity-demonstrating the advantages this method provides.

\section{REFERENCES}

Arnett, W. D. 1973, in Explosive Nucleosynthesis, ed. D. N. Schramm and W. D. Arnett (Austin: University of Texas Press).

Arnett, W. D., and Bowers, R. L. 1974, Neutron Star Structure -A Survey, Report of the Nuclear and Relativistic Astrophysics Group of the University of Texas at Austin, unpublished.

Arnett, W. D., and Schramm, D. N. 1973, Ap. J. (Letters), 184, L47.

Baym, G., and Pethick, C. 1975, Ann. Rev. Nucl. Sci., 25, 82.

Blake, J. B., and Schramm, D. N. 1973, Ap. J., 179, 569.

Bludman, S. A., and Ruderman, M. A. 1975, Ap. J. (Letters), 195, L19.

Blumenthal, G. R., and Tucker, W. H. 1974, Ann. Rev. Astr. Ap., 12, 23.

Burbidge, E. M., Burbidge, G. R., Fowler, W. A., and Hoyle, F. 1957, Rev. Mod. Phys., 29, 547.

Chandrasekhar, S. 1969, Ellipsoidal Figures of Equilibrium (New Haven: Yale University Press).

Cheng, A. 1974, Ap. and Space Sci., 31, 49.

Corinaldesi, E., and Papapetrou, A. 1951, Proc. R. Soc. London $A, 209,259$.

De Loore, C., De Greve, J. P., van den Heuvel, E. P. J., and De Cuyper, J. P. 1974, Proc. 2nd IAU Regional Meeting, Trieste.

Epstein, R. I., Lattimer, J. M., and Schramm, D. N. 1976, submitted to Nature.

Fishbone, L. G. 1972, Ap. J. (Letters), 175, L155.

Flannery, B. P., and van den Heuvel, E. P. J. 1975, Astr. and Ap., 39, 61.

Hainebach, K. L., Norman, E. B., and Schramm, D. N. 1976, Ap. J., 203, 245.

Heintz, W. D. 1969, Proc. R. Soc. Canada, 63, 275.

Hill, R. N. 1974, J. Math. Phys., 15, 1596.
Hulse, R. A., and Taylor, J. H. 1975, Ap. J. (Letters), 195, L51.

Iben, I. 1967, Ann. Rev. Astr. Ap., 5, 571.

Kippenhahn, R., and Weigert, A. 1967, Zs. f. Ap., 65, 251.

Landau, L. D., and Lifshitz, E. M. 1971, The Classical Theory of Fields (3d rev. English ed.; London: Pergamon Press).

Lande, K., Bozoki, G., Frati, W., Lee, C. K., Fenyves, E., and Saavedra, O. 1974, Nature, 251, 485.

Lattimer, J. M. 1976, unpublished Ph.D. thesis, University of Texas at Austin.

Lattimer, J. M., Mackie, F. D., Ravenhall, D. G., and Schramm, D. N. 1975, Bull. AAS, 7, 546.

Lattimer, J. M., and Schramm, D. N. 1974, Ap. J. (Letters), 192, L145 (LS).

Mashhoon, B. 1972, unpublished Ph.D. thesis, Princeton University.

- 1975, Ap.J., 197, 205.

Matzner, R. A., and Nutku, Y. 1974, Proc. R. Soc. London A, 336, 285.

Milgrom, M., and Salpeter, E. E. 1975, Ap. J., 196, 589.

Miller, B. E. 1974, Ap. J., 187, 609.

Misner, C. W., Thorne, K. S., and Wheeler, J. A. 1973, Gravitation (San Francisco: Freeman).

Morton, D. C. 1960, Ap. J., 133, 146.

Oort, S. H. 1965, in Stars and Stellar Systems, Vol. 5, ed. A. Blaauw and M. Schmidt (Chicago: University of Chicago Press), chap. 21.

Ostriker, J. P., Richstone, D. O., and Thuan, T. X. 1974, Ap. J. (Letters), 188, L87.

Paczynski, B. 1971, Ann. Rev. Astr. Ap., 9, 813.

. 1974, Astr. and Ap., 34, 161.

. 1976, private communication.

Page, D. N., and Thorne, K. S. 1974, Ap. J., 191, 507.

Pirani, F. A. E. 1956, Acta Phys. Polon., 15, 399.

Plavec, M. 1968, Adv. Astr. Ap., 6, 201. 
Press, W. H., and Teukolsky, S. A. 1973, Ap. J., 181, 513.

Roberts, D. H., Masters, A. R., and Arnett, W. D. 1976, Ap. J. (Letters), 203, 196.

Roberts, P. H. 1962, Ap. J., 136, 1108.

Rogerson, J. B., and York, D. G. 1974, Ap. J. (Letters), 186, L95.

Rossner, L. F. 1967, Ap. J., 149, 145.

Schramm, D. N., and Arnett, W. D. 1975, Ap. J., 198, 629.

Shakura, N. I., and Sunyaev, R. A. 1973, Astr. and Ap., 24 337.

Smarr, L. 1974, unpublished Ph.D. thesis, University of Texas at Austin.
Tinsley, B. 1975, Pub. A.S.P., 87, 837.

van den Heuvel, E. P. J. 1975, Ap. J. (Letters), 198, L109.

van den Heuvel, E. P. J., and de Loore, C. 1973, Astr. and Ap., $25,387$.

Wheeler, J. A. 1971, Pontificae Acad. Sci. Scripta Varia, 35, 539.

Wheeler, J. C., McKee, C. F., and Lecar, M. 1974, Ap. J. (Letters), 192, L71.

Zel'dovich, Ya. B., Ivanova, I. N., and Nedezin, D. K. 1972 Soviet Astr.-AJ, 16, 209.

Zytkov, A., and Thorne, K. S. 1974, report presented at the Workshop on Compact X-ray Sources, Trieste.

JAMES M. LATTimer: Department of Astronomy, University of Illinois, Urbana, IL 61801

DAVID N. SCHRAMm: Enrico Fermi Institute, Laboratory for Space Research, 933 East 56th St., Chicago, IL 60637 